\title{
Categorification of Persistent Homology
}

\author{
Peter Bubenik · Jonathan A. Scott
}

Received: 8 March 2013 / Revised: 12 November 2013 / Accepted: 10 January 2014 /

Published online: 28 January 2014

(C) Springer Science+Business Media New York 2014

\begin{abstract}
We redevelop persistent homology (topological persistence) from a categorical point of view. The main objects of study are $(\mathbb{R}, \leq)$-indexed diagrams in some target category. A set of such diagrams has an interleaving distance, which we show generalizes the previously studied bottleneck distance. To illustrate the utility of this approach, we generalize previous stability results for persistence, extended persistence, and kernel, image, and cokernel persistence. We give a natural construction of a category of $\varepsilon$-interleavings of $(\mathbb{R}, \leq)$-indexed diagrams in some target category and show that if the target category is abelian, so is this category of interleavings.
\end{abstract}

Keywords Applied topology · Persistent topology · Topological persistence · Diagrams indexed by the poset of real numbers - Interleaving distance

\section{Introduction}

The ideas of topological persistence [13] and persistent homology [21] have had a great impact on computational geometry and the newer field of applied topology. This method applies geometric and algebraic constructions to input from applications, followed by clever modifications of tools from algebraic topology. It has found many uses, and the results can be global qualitative descriptions inaccessible to other methods. Subsequent theoretical work in this subject has given stronger results and adapted the basic constructions so that they might be applied in more diverse situations. For surveys and books on this subject, see [3, 11, 12, 15, 20].

\footnotetext{
P. Bubenik - J.A. Scott

Department of Mathematics, Cleveland State University, 2121 Euclid Ave., RT 1515, Cleveland,

$\mathrm{OH} 44115$, USA

e-mail: j.a.scott3@csuohio.edu

P. Bubenik

e-mail: p.bubenik@csuohio.edu
} 


\subsection{Motivation}

Throughout its history, algebraic topology has frequently undergone a process in which previous results were redeveloped from a more abstract point of view. This has had two main advantages. First, abstraction clarified the key ideas and proofs. Second, and more importantly, the more abstract setting allowed previous results to be vastly generalized and applied in ways never considered in the original. The development and use of category theory has been a critical part of this process.

The main motivation of this paper is to subject the ideas and results of topological persistence to this process.

\subsection{Prior Work}

In the descriptions below, we will make anachronistic use of this paper's point of view, in particular, its focus on diagrams (see (1) and Sect. 2.1).

Two foundational papers in this subject are [13] and [21]. In the first, Edelsbrunner, Letscher, and Zomorodian define persistent homology for $\left(\mathbb{Z}_{+}, \leq\right)$-indexed diagrams of finite-dimensional vector spaces that are obtained from filtered finite simplicial complexes by taking simplicial homology with coefficients in a field. In the second, Zomorodian and Carlsson take a purely algebraic point of view. They define persistent homology for tame $\left(\mathbb{Z}_{+}, \leq\right)$-indexed diagrams of finite-dimensional vector spaces and prove a bijection between isomorphism classes of such tame diagrams and finite barcodes whose endpoints lie in $\mathbb{Z}_{+} \cup\{\infty\}$. ( $\left.\mathbb{Z}_{+}, \leq\right)$-indexed diagrams of finite-dimensional vector spaces are called persistence modules.

These papers are rounded out by [7], where Cohen-Steiner, Edelsbrunner, and Harer prove that persistent homology is useful in applications by showing that it is stable in the following sense. Let $f, g: X \rightarrow \mathbb{R}$ be continuous functions on a triangulable space. Define an $(\mathbb{R}, \leq)$-indexed diagram of topological spaces, $F$, by setting $F(a)=f^{-1}(-\infty, a]$ and letting $F(a \leq b)$ be given by inclusion. Define $G$ similarly using $g$. Let $H$ be the singular homology functor with coefficients in a field. Assume that $H F$ and $H G$ are diagrams of finite-dimensional vector spaces and that they are tame. Then the bottleneck distance between $H F$ and $H G$ is bounded by the supremum norm between $f$ and $g$.

This stability result is significantly strengthened by Chazal, Cohen-Steiner, Glisse, Guibas, and Oudot in [5]. They drop the assumptions that $X$ be triangulable, that $f, g$ be continuous, and that $H F$ and $H G$ be tame. Their approach is crucial to this paper. They explicitly work with $(\mathbb{R}, \leq)$-indexed diagrams, though they consider them from an algebraic, not categorical, point of view. They define the interleaving distance, $d$, between such diagrams, and define the bottleneck distance, $d_{B}$, between such diagrams using limits of discretizations, and show that $d_{B} \leq d$.

The basic idea of persistent homology has been extended in numerous ways. Here we focus on two particularly useful extensions, given in [8] and [9]. In the first, Cohen-Steiner, Edelsbrunner, and Harer define extended persistence for finitedimensional simplicial complexes with a finite filtration and homology with coefficients in $\mathbb{Z} / 2 \mathbb{Z}$. They show that in this case the stability result of [7] applies. In the second, Cohen-Steiner, Edelsbrunner, Harer, and Morozov consider a triangulated 
space $X$ with subcomplex $Y$ and maps $f, f^{\prime}: X \rightarrow \mathbb{R}$ and $g, g^{\prime}: Y \rightarrow \mathbb{R}$ such that for all $y \in Y, f(y) \leq g(y)$ and $f^{\prime}(y) \leq g^{\prime}(y)$. They assume that $f, f^{\prime}, g, g^{\prime}$ are continuous and tame. Then there are maps of the corresponding $(\mathbb{R}, \leq)$-indexed diagrams $H G \rightarrow H F$ and $H G^{\prime} \rightarrow H F^{\prime}$. Let $\varepsilon=\max \left\{\left\|f-f^{\prime}\right\|_{\infty},\left\|g-g^{\prime}\right\|_{\infty}\right\}$. The authors show that the bottleneck distances between the kernels, images, and cokernels, respectively, of these maps are each bounded above by $\varepsilon$.

An early categorical approach to persistence can be found in [2].

\subsection{Our Contributions}

We redevelop persistent homology from a categorical point of view. In particular, we consider diagrams indexed by $(\mathbb{R}, \leq)$ to be the main objects of study. An $(\mathbb{R}, \leq)$ indexed diagram consists of a set of objects $X(a)$ for each $a \in \mathbb{R}$ and morphisms

$$
X(a) \rightarrow X(b)
$$

for each $a \leq b$, satisfying certain composition and unit axioms (see Sect. 2.1). The objects and morphisms lie in some fixed category, such as topological spaces and continuous maps, or finite-dimensional vector spaces and linear transformations. In Sect. 2.2, we show that the basic constructions of persistent homology are special cases of this construction. We will show that in this setting, functoriality provides concise and powerful results.

In Sect. 3, we define an $\varepsilon$-interleaving for $(\mathbb{R}, \leq$ )-indexed diagrams (Definition 3.1) and show that this induces a metric (Theorem 3.3 and Corollary 3.5).

We specialize to $(\mathbb{R}, \leq)$-indexed diagrams of finite-dimensional vector spaces in Sect. 4. These are also called (real) persistence modules. We study barcodes, persistence diagrams, and the bottleneck and interleaving distances. We define finite type diagrams to be direct sums of certain indecomposable diagrams (Definition 4.1). We show that these are exactly the tame diagrams (Theorem 4.6). Furthermore, we show that they satisfy a Krull-(Remak-)Schmidt theorem. That is, the direct sum decomposition is essentially unique (Corollary 4.7). We show that the metric space of finite barcodes together with the bottleneck distance embeds isometrically into the metric space of $(\mathbb{R}, \leq)$-indexed diagrams of finite-dimensional vector spaces with the interleaving distance (Theorem 4.16). This result justifies our assertion that our stability theorems, which use the interleaving distance, are generalizations of previously established stability theorems, which use the bottleneck distance.

In Sect. 5, we give a simple formal argument for a stability theorem for the interleaving distance. By the previous work identifying the interleaving and bottleneck distances, this allows us to both remove assumptions and to significantly generalize, the stability result of [7]. Given any functions $f, g: X \rightarrow \mathbb{R}$ on any topological space $X$ and any functor $H$ on topological spaces, we show that the interleaving distance of $H F$ and $H G$ is bounded above by the supremum norm between $f$ and $g$ (Theorem 5.1).

We generalize the extended persistence construction of [8] in Sect. 6. For any (not necessarily continuous) map $f: X \rightarrow(-\infty, M] \subset \mathbb{R}$, we define an $(\mathbb{R}, \leq)$-indexed diagram of pairs of topological spaces. We prove a stability theorem for extended persistence. Given $f, g: X \rightarrow(-\infty, M]$ and corresponding diagrams $F$ and $G$ of 
pairs of spaces, and any functor $H$ on pairs of spaces, the interleaving distance between $H F$ and $H G$ is bounded above by the supremum norm between $f$ and $g$ (Theorem 6.1).

In Sect. 7, we define a category of interleavings of $(\mathbb{R}, \leq)$-indexed diagrams in a given base category (Definition 7.1). We show that in the case that the base category is an abelian category, then so is this category of interleavings (Theorem 7.10). As a result, this category has direct sums, kernels, images, and cokernels. As an application, we generalize the stability theorem of [9], dropping the assumptions that $X$ and $Y$ are triangulated, that $f, f^{\prime}, g, g^{\prime}$ are continuous and tame, replacing the subcomplex condition with a continuous map $Y \rightarrow X$ and replacing singular homology with coefficients in $\mathbb{Z} / 2 \mathbb{Z}$, with any functor from topological spaces to an abelian category (Theorem 7.13). We also give a version of this theorem for extended persistence (Theorem 7.14).

\subsection{Comparison with Other Recent Work}

The material in Sect. 4 has been studied in greater detail in the algebraic setting by Lesnick [17] and by Chazal, de Silva, Glisse, and Oudot [6]. In particular, Lesnick proves a more general Isometry Theorem [17, Theorem 2.4.2], removing the condition that the persistence modules have finite type from our Theorem 4.16. This is further generalized to q-tame persistence modules in [6, Theorem 4.11]. We also remark that one of the directions in our isometry theorem is due to [5]. Crawley-Boevey [10] has shown that any $(\mathbb{R}, \leq)$-indexed diagram of finite-dimensional vector spaces is a direct sum of interval modules. In light of this result, it may be possible to generalize some of our work in Sect. 4.

Our Stability Theorem (Theorem 5.1) is quite general and once the categorical machinery has been set up, has a very simple proof. However it applies to persistence modules, not for their corresponding persistence diagrams. In the language of [1], it is a soft stability theorem. Hard stability theorems [5-7] giving stability for persistence diagrams require more detailed analysis. For example, an Isometry Theorem can be used to show that soft stability implies hard stability. On the other hand, our Stability Theorem is more general in that it applies to functors to arbitrary categories. For a simple example, consider homology with integer coefficients. It also clarifies what part of stability is purely formal and what part requires detailed analysis. This viewpoint is expanded upon in [1].

\section{Background}

In Sect. 2.1, we give the basic definitions of category theory that we will use throughout the paper. In Sect. 2.2, we show how the standard constructions of persistent homology fit within our categorical approach. The last two sections give more specialized background. In Sect. 2.3, we define abelian categories, which we use in Sect. 7. In Sect. 2.4, we give some algebraic definitions used in the proof of Theorem 4.6. 


\subsection{Categorical Terminology}

A category, $\mathbf{C}$, consists of a class of objects, $\mathbf{C}_{0}$, and for each pair of objects $X, Y \in$ $\mathbf{C}_{0}$, a set of morphisms, $\mathbf{C}(X, Y)$. We often write $f: X \rightarrow Y$ if $f \in \mathbf{C}(X, Y)$. For every triple $X, Y, Z \in \mathbf{C}_{0}$, there is a set mapping,

$$
\mathbf{C}(Y, Z) \times \mathbf{C}(X, Y) \rightarrow \mathbf{C}(X, Z), \quad(g, f) \mapsto g f,
$$

called composition. Composition must be associative, in the sense that $(h g) f=$ $h(g f)$. Finally, for all $X \in \mathbf{C}$, there is an identity morphism, $\operatorname{Id}_{X}: X \rightarrow X$, that satisfies $\operatorname{Id}_{X} f=f$ and $g \operatorname{Id}_{X}=g$ for all $f: W \rightarrow X$ and all $g: X \rightarrow Y$. The identity morphism is unique. We will regularly abuse notation and write $X \in \mathbf{C}$ to mean $X \in \mathbf{C}_{0}$.

A category $\mathbf{C}$ is called small if $\mathbf{C}_{0}$ is a set rather than a proper class.

Example 2.1 Let Top be the category whose objects are all topological spaces and whose morphisms are all continuous maps. Here, composition is the composition of mappings, and the identity morphisms are what one would expect.

A related category is Pair, whose objects are pairs $(X, A)$, where $X$ is a topological space, and $A$ is a subspace of $X$. A morphism from $(X, A)$ to $(Y, B)$ is a continuous map $f: X \rightarrow Y$ such that $f(A) \subset B$. We express this condition by saying that the diagram

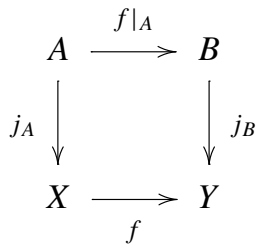

commutes, where $j_{A}$ and $j_{B}$ are the canonical inclusions, and $\left.f\right|_{A}$ is $f$ restricted to $A$.

Example 2.2 Let Vec be the category of finite-dimensional vector spaces over a fixed ground field $\mathbb{F}$, along with the linear transformations between them. Again, composition is that of mappings, and the identities are simply the identity mappings.

A graded vector space is a collection $V_{*}=\left\{V_{n}\right\}_{n \in \mathbb{Z}}$ with each $V_{n} \in$ Vec. A morphism, $f_{*}: V_{*} \rightarrow W_{*}$, of graded vector spaces is a sequence, $f_{*}=\left\{f_{n}: V_{n} \rightarrow W_{n}\right\}$. Denote by grVec the category of graded vector spaces and their morphisms.

A reflexive, antisymmetric, and transitive relation $\leq$ on a set $P$ is called a partial order. A set $P$ equipped with a partial order is called a poset. We identify each poset $P$ with the small category $\mathbf{P}$ that has $\mathbf{P}_{0}=P$, and $\mathbf{P}(x, y)$ has precisely one element if $x \leq y$ and is otherwise empty. Conversely, let $\mathbf{P}$ be a small category in which each set of morphisms contains at most one element, and if $\mathbf{P}(x, y)$ and $\mathbf{P}(y, x)$ are both nonempty, then $x=y$. Then $\mathbf{P}_{0}$ is a poset, with partial ordering defined by $x \leq y$ if and only if $\mathbf{P}(x, y) \neq \varnothing$. 
Example 2.3 The set of real numbers, $\mathbb{R}$, with its usual ordering is a poset. The set of integers, $\mathbb{Z}$, of nonnegative integers $\mathbb{Z}_{+}$, and $[\mathbf{n}]=\{0, \ldots, n\}$, are subposets. For a partial order that is not a total order, consider the set $\mathbb{R}^{n}$ with $n>1$ and the ordering $\left(x_{1}, \ldots, x_{n}\right) \leq\left(y_{1}, \ldots, y_{n}\right)$ if and only if $x_{i} \leq y_{i}$ for all $i=1, \ldots, n$.

Two objects $X, Y \in \mathbf{C}_{0}$ are said to be isomorphic if there exist morphisms $f: X \rightarrow Y$ and $g: Y \rightarrow X$ such that $g f=\operatorname{Id}_{X}$ and $f g=\operatorname{Id}_{Y}$. In this case, $f$ and $g$ are called isomorphisms. Clearly, isomorphism is an equivalence relation. In Top, isomorphism becomes homeomorphism.

The notion of functor expresses relationships between categories. Let $\mathbf{A}$ and $\mathbf{C}$ be categories. A functor, $F: \mathbf{A} \rightarrow \mathbf{C}$, consists of a mapping $F: \mathbf{A}_{0} \rightarrow \mathbf{C}_{0}$, and for each pair $X, Y \in \mathbf{A}_{0}$, a mapping $F: \mathbf{A}(X, Y) \rightarrow \mathbf{C}(F(X), F(Y))$. These mappings must be compatible with the composition and identity structure of the categories, in the sense that if $f: X \rightarrow Y$ and $g: Y \rightarrow Z$, then $F(g f)=F(g) F(f)$, and if $X \in \mathbf{A}_{0}$, then $F\left(\operatorname{Id}_{X}\right)=\operatorname{Id}_{F(X)}$.

Example 2.4 Denote by $H_{*}(-)$ singular homology with coefficients in some fixed field, $\mathbb{F}$. Then $H_{*}(X)$ is a graded $\mathbb{F}$-vector space for all $X \in \mathbf{T o p}_{0}$. Furthermore, if $f: X \rightarrow Y$ is continuous, then we get the induced homomorphism, $H_{*}(f)$ : $H_{*}(X) \rightarrow H_{*}(Y)$. Since $H_{*}(g f)=H_{*}(g) H_{*}(f)$, singular homology defines a functor $H_{*}:$ Top $\rightarrow$ grVec. If we consider only homology in degree $k$, then we get a functor $H_{k}:$ Top $\rightarrow$ Vec.

Let $F, G: \mathbf{A} \rightarrow \mathbf{C}$ be functors. A natural transformation $\eta: F \Rightarrow G$ consists of, for all $A \in \mathbf{A}_{0}$, a morphism $\eta_{A}: F(A) \rightarrow G(A)$ in $\mathbf{C}$ such that whenever $\varphi: A \rightarrow A^{\prime}$ is a morphism, the diagram

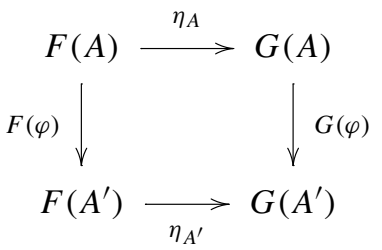

commutes. If for all $A \in \mathbf{A}_{0}, \eta_{A}$ is an isomorphism, then $\eta$ is called a natural isomorphism, and we write $F \cong G$.

Example 2.5 Consider the poset $(\mathbb{R}, \leq)$, and let $\varepsilon \geq 0$. Define $T_{\varepsilon}:(\mathbb{R}, \leq) \rightarrow(\mathbb{R}, \leq)$ by $T_{\varepsilon}(x)=x+\varepsilon$. If $x \leq y$, then $x+\varepsilon \leq y+\varepsilon$, so $T_{\varepsilon}$ defines a functor to $(\mathbb{R}, \leq)$ to itself. We call $T_{\varepsilon}$ translation by $\varepsilon$. Since $\varepsilon \geq 0$ and $x \leq x+\varepsilon$ for all $x \in \mathbb{R}$, we get a natural transformation $\eta: I \Rightarrow T_{\varepsilon}$, where $I: \mathbb{R} \rightarrow \mathbb{R}$ is the identity functor.

The collection of all small categories, and the functors between them, itself forms a category, denoted by Cat.

Let $\mathbf{C}$ and $\mathbf{D}$ be categories with $\mathbf{C}$ small. A functor, $F: \mathbf{C} \rightarrow \mathbf{D}$, is called a diagram in $\mathbf{D}$ indexed by $\mathbf{C}$. The collection of all such functors, and natural transformations between them, forms a category, $\mathbf{D}^{\mathbf{C}}$. 
Example 2.6 Let $\mathbf{C}$ be the discrete category whose objects are the integers; the only morphisms are the identity morphisms. Then $\mathbf{V e c}^{\mathbf{C}}=$ grVec.

Example 2.7 A diagram $F$ in a category $\mathbf{D}$ indexed by $\left(\mathbb{Z}_{+}, \leq\right)$is a sequence of morphisms in D:

$$
F(0) \rightarrow F(1) \rightarrow F(2) \rightarrow \cdots .
$$

If $\mathbf{D}=$ Top, then each $F(n)$ is a topological space, and the morphisms are continuous maps. If $\mathbf{D}=\mathbf{V e c}$, then each $F(n)$ is a finite-dimensional vector space, and the morphisms are linear maps.

Indexed by $(\mathbb{Z}, \leq)$, the diagram extends in both directions:

$$
\cdots \rightarrow F(-2) \rightarrow F(-1) \rightarrow F(0) \rightarrow F(1) \rightarrow F(2) \rightarrow \cdots .
$$

If the indexing category is $(\mathbb{R}, \leq)$, then we have objects $F(a)$ for all $a \in \mathbb{R}$, and for each $a \leq b$, a morphism $F(a) \rightarrow F(b)$.

Given two natural transformations $\varphi: F \Rightarrow G$ and $\psi: G \Rightarrow H$, their (vertical) composition $\psi \circ \varphi$ is the natural transformation given by the composition of morphisms $F(A) \stackrel{\varphi_{A}}{\longrightarrow} G(A) \stackrel{\psi_{A}}{\longrightarrow} H(A)$ and the composition of the corresponding commutative squares (2).

For $i=1,2$, let $F_{i}, G_{i}: \mathbf{A}_{i} \rightarrow \mathbf{A}_{i+1}$ be functors, and let $\varphi_{i}: F_{i} \Rightarrow G_{i}$ be a natural transformation. The (horizontal) composition of $\varphi_{1}$ and $\varphi_{2}$ is the natural transformation, $\varphi_{2} \varphi_{1}: F_{2} F_{1} \Rightarrow G_{2} G_{1}$, defined on morphisms by $\left(\varphi_{2} \varphi_{1}\right)(f)=\varphi_{2}\left(\varphi_{1}(f)\right)$. For every functor $H$, there is the identity natural isomorphism $\operatorname{Id}_{H}: H \Rightarrow H$. We abuse notation and refer to the horizontal composition of a natural transformation $\varphi$ with $\operatorname{Id}_{H}$ as the composition of $\varphi$ with $H$.

\subsection{Categorical Persistent Homology}

In this section, we consider two prototypical examples in which persistent homology is applied and show how they fit into our categorical framework. We also show how diagrams indexed by $[\mathbf{n}],\left(\mathbb{Z}_{+}, \leq\right)$, and $(\mathbb{Z}, \leq)$ are special cases of diagrams indexed by $(\mathbb{R}, \leq)$. Finally, we define persistent homology.

\subsubsection{Filtered Simplicial Complexes}

First, let $K$ be a finite simplicial complex with filtration

$$
\emptyset=K_{0} \subseteq K_{1} \subseteq \cdots \subseteq K_{n}=K .
$$

Then this gives an [n]-indexed diagram of topological spaces, i.e., $K \in \mathbf{T o p}^{[\mathbf{n}]}$, with $K(i)=K_{i}$ and $K(i \leq j)$ given by inclusion.

Let $H_{k}$ be the degree $k$ simplicial homology functor with coefficients in a field $\mathbb{F}$. Then $H_{k} K$ is an [n]-indexed diagram of finite-dimensional vector spaces. That is, $H_{k} K(i)=H_{k}\left(K_{i}, \mathbb{F}\right)$ and $H_{k} K(i \leq j)$ is the map induced on homology by the inclusion $K_{i} \hookrightarrow K_{j}$. So $H_{k} K \in \mathbf{V e c}^{[\mathbf{n}]}$.

We can sum homology in all degrees to get $H F \in \operatorname{Vec}^{[\mathbf{n}]}$, given by $H F(i)=$ $\bigoplus_{k} H_{k}\left(K_{i}, \mathbb{F}\right)$. 


\subsubsection{Sublevel Sets}

Second, let $X$ be a topological space, and let $f: X \rightarrow \mathbb{R}$ be a not necessarily continuous real-valued function on $X$. Let $a \in \mathbb{R}$. We consider the sublevel set (or lower excursion set, also called a half space)

$$
f^{-1}((-\infty, a])=\{x \in X \mid f(x) \leq a\} .
$$

For simplicity, we will usually write $f^{-1}(-\infty, a]$. We consider it as a topological space using the subspace topology. Notice that if $a \leq b$, then $f^{-1}(-\infty, a] \subseteq$ $f^{-1}(-\infty, b]$, and this inclusion is a continuous map.

This data can be assembled into an $(\mathbb{R}, \leq)$-indexed diagram of topological spaces, $F \in \operatorname{Top}^{(\mathbb{R}, \leq)}$. For $a \in \mathbb{R}$, we define $F(a)=f^{-1}(-\infty, a]$. For $a \leq b$, we define $F(a \leq b)$ to be the inclusion $f^{-1}(-\infty, a] \hookrightarrow f^{-1}(-\infty, b]$. It is easy to check that this defines a functor $F:(\mathbb{R}, \leq) \rightarrow$ Top.

Let $H_{k}$ be the $k$ th singular homology functor with coefficients in some field $\mathbb{F}$. Then $H_{k} F$ is an $(\mathbb{R}, \leq)$-indexed diagram of (not necessarily finite-dimensional) vector spaces. That is, $H_{k} F(a)=H_{k}\left(f^{-1}(-\infty, a], \mathbb{F}\right)$, and for $a \leq b, H_{k} F(a \leq b)$ is the map induced on homology by the inclusion $f^{-1}(-\infty, a] \hookrightarrow f^{-1}(-\infty, b]$. If $f$ has the property that for all $a \in \mathbb{R}, H_{k}\left(f^{-1}(-\infty, a], \mathbb{F}\right)$ is a finite-dimensional vector space, then $H_{k} F \in \mathbf{V e c}^{(\mathbb{R}, \leq)}$.

If $f$ has the property that for all $a \in \mathbb{R}, H_{*}\left(f^{-1}(\infty, a], \mathbb{F}\right)$ is finite-dimensional, then $H F \in \mathbf{V e c}^{(\mathbb{R}, \leq)}$ is given by $H F(a)=\bigoplus_{k} H_{k}\left(f^{-1}(-\infty, a], \mathbb{F}\right)$.

\subsubsection{Diagrams by $[\mathbf{n}],\left(\mathbb{Z}_{+}, \leq\right)$, and $(\mathbb{Z}, \leq)$}

In this paper, we will only consider the indexing category $(\mathbb{R}, \leq)$. However, this case also includes the cases $[\mathbf{n}],\left(\mathbb{Z}_{+}, \leq\right)$, and $(\mathbb{Z}, \leq)$, by the following observation. Consider $F \in \mathbf{T o p}^{[\mathbf{n}]}$. Then we can extend $F$ to an $(\mathbb{R}, \leq)$-indexed diagram as follows. The inclusion functor $\mathbf{i}:[\mathbf{n}] \rightarrow(\mathbb{R}, \leq)$ given by $\mathbf{i}(j)=j$ has a retraction functor $\mathbf{r}:(\mathbb{R}, \leq) \rightarrow[\mathbf{n}]$ given by

$$
\mathbf{r}(a)= \begin{cases}0 & \text { if } a \leq 0, \\ \lfloor a\rfloor & \text { if } 0<a<n, \\ n & \text { if } a \geq n\end{cases}
$$

Thus, the composite functor $F \mathbf{r}$ is an element of $\mathbf{T o p}^{(\mathbb{R}, \leq)}$, and $F \mathbf{r i}=F$. There are similarly defined retraction functors to $\left(\mathbb{Z}_{+}, \leq\right)$and to $(\mathbb{Z}, \leq)$.

\subsubsection{Persistent Homology}

Given a diagram $F \in \mathbf{T o p}^{(\mathbb{R}, \leq)}$, we define the p-persistent kth homology group of $F(a)$ to be the image of the map $H_{k} F(a \leq a+p)$.

\subsubsection{Persistence Modules}

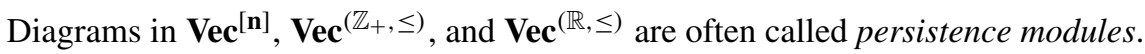




\subsection{Abelian Categories}

In this section, we recall standard definitions from category theory that we will use in Sect. 7. Details can be found in, for example, [18]. Throughout this section, $\mathbf{C}$ denotes a category.

\subsubsection{Initial, Terminal, and Final Objects}

We say that an object $\varnothing$ of $\mathbf{C}$ is initial if, for every object $X$ in $\mathbf{C}$, there is a unique morphism $\varnothing \rightarrow X$. An object $*$ is terminal if, for every object $X$, there is a unique morphism $X \rightarrow *$. It follows from these definitions that initial and terminal objects, if they exist, are unique up to canonical isomorphism. If an object is both initial and terminal, we say that it is zero and denote it by 0 . In the presence of a zero object, for every pair of objects $X, Y \in \mathbf{C}$, we can define the zero morphism $0: X \rightarrow Y$ to be the composite of the unique morphisms, $X \rightarrow 0 \rightarrow Y$. It follows by uniqueness that if $f$ is any morphism, then $f 0=0 f=0$.

\subsubsection{Monomorphisms, Epimorphisms, Kernels, and Cokernels}

Let $f: X \rightarrow Y$ be a morphism. We say that $f$ is a monomorphism if, whenever $g, h: W \rightarrow X$ are morphisms such that $f g=f h$, we have that $g=h$. Dually, $f$ is an epimorphism if, whenever $k, \ell: Y \rightarrow Z$ are morphisms such that $k f=\ell f$, then $k=\ell$. An isomorphism class of monomorphisms to $Y$ is called a subobject of $Y$. Dually, isomorphism classes of epimorphisms are called quotient objects.

Suppose that $\mathbf{C}$ has a zero object, 0 . Let $f: X \rightarrow Y$ be a morphism in $\mathbf{C}$. The kernel of $f$ is the equalizer of $f$ and $0: X \rightarrow Y$. That is, the kernel is a morphism, $j: \operatorname{ker} f \rightarrow X$, such that $f j=0$ and that is "universal" in the sense that whenever $g$ : $W \rightarrow X$ is a morphism satisfying $f g=0$, then there is a unique morphism $\tilde{g}: W \rightarrow$ ker $f$ such that $j \tilde{g}=g$. Since $j$ is an equalizer, it follows that $j$ is a monomorphism. So ker $f$ represents a subobject of $X$. Thus, the kernel is the appropriate categorical notion for the part of $X$ that $f$ sends to 0 . We use the word "kernel" to mean both the object ker $f$ and the universal morphism, $\operatorname{ker} f \rightarrow X$, according to the context. We remark that it follows from the definition that all such universal objects are unique up to unique isomorphism. That is, if $g: W \rightarrow X$ and $g^{\prime}: W^{\prime} \rightarrow X$ are both kernels of $f: X \rightarrow Y$, then there is a unique isomorphism $\tilde{g}: W \rightarrow W^{\prime}$ such that $g^{\prime} \tilde{g}=g$.

Dually, the cokernel of $f: X \rightarrow Y$ is the coequalizer of $f$ and 0 . That is, the cokernel is a universal morphism $q: Y \rightarrow \operatorname{coker} f$, such that whenever $h: Y \rightarrow Z$ satisfies $h f=0$, there exists a unique morphism $\tilde{h}: \operatorname{coker} f \rightarrow Z$ such that $\tilde{h} q=h$. Again, we sometimes abuse notation and use "cokernel" to refer to the object, coker $f$. Since $q$ is a coequalizer, it is an epimorphism, and coker $f$ represents a quotient object of $Y$. Again, cokernels, if they exist, are unique up to canonical isomorphism.

\subsubsection{Products, Coproducts, Pull-Backs, and Push-Outs}

Let $X, Y \in \mathbf{C}$. The product of $X$ and $Y$, if it exists in $\mathbf{C}$, is an object denoted by $X \times Y$, along with morphisms $p_{X}: X \times Y \rightarrow X$ and $p_{Y}: X \times Y \rightarrow Y$ satisfying the 
following universal property. For every object $W$ together with a pair of morphisms $f_{X}: W \rightarrow X$ and $f_{Y}: W \rightarrow Y$, there is a unique morphism $f: W \rightarrow X \times Y$ such that $f_{X}=p_{X} f$ and $f_{Y}=p_{Y} f$. The product, if it exists, is unique up to canonical isomorphism.

Dually, the coproduct of $X$ and $Y$, if it exists in $\mathbf{C}$, is an object $X \oplus Y$, along with morphisms $j_{X}: X \rightarrow X \oplus Y$ and $j_{Y}: Y \rightarrow X \oplus Y$ satisfying the following universal property. For every object $U$ together with a pair of morphisms $g_{X}: X \rightarrow U$ and $g_{Y}: Y \rightarrow U$, there is a unique morphism $g: X \oplus Y \rightarrow U$ such that $g_{X}=$ $g j_{X}$ and $g_{Y}=g j_{Y}$. The coproduct, if it exists, is unique up to canonical isomorphism.

Consider the diagram $X \stackrel{f}{\rightarrow} Z \stackrel{g}{\leftarrow} Y$. The pull-back of $f$ and $g$ consists of an object $P$ and morphisms $X \stackrel{p_{X}}{\longleftarrow} P \stackrel{p_{Y}}{\longrightarrow} Y$ satisfying $f p_{X}=g p_{Y}$ and the following universal property. For each diagram

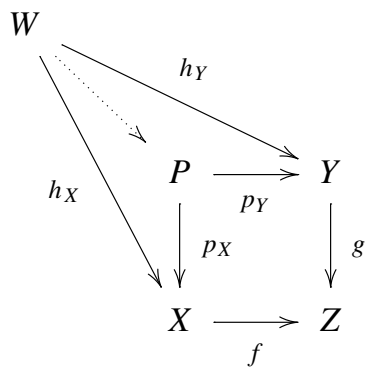

where the outer paths commute, there is a unique morphism $W \rightarrow P$ that makes the entire diagram commute. The pull-back is unique up to canonical isomorphism and is denoted by $P=X \times{ }_{Z} Y$ when there can be no ambiguity concerning $f$ and $g$.

Dually, the push-out of the diagram $Y \stackrel{f}{\leftarrow} X \stackrel{g}{\rightarrow} Z$ consists of an object $Q$ along with universal morphisms $Y \stackrel{j_{Y}}{\longrightarrow} Q \stackrel{j_{Z}}{\longleftarrow} Z$ satisfying $j_{Y} f=j_{Z} g$ and the following universal property. Whenever the outer paths in the diagram

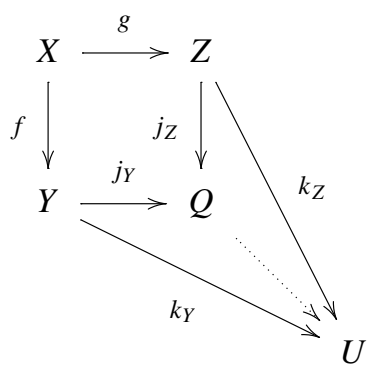

commute, there is a unique morphism $k: Q \rightarrow U$ making the entire diagram commute. The push-out is unique up to canonical isomorphism, and is denoted by $Q=Y \bigoplus_{X} Z$. 


\subsubsection{Abelian Categories}

An abelian category is a category that contains a zero object and all products and coproducts, in which every morphism has a kernel and cokernel, every monomorphism is a kernel, and every epimorphism is a cokernel. By Freyd [14], every abelian category, $\mathbf{A}$, is preadditive, that is, it is naturally enriched in abelian groups. This means that for all pairs of objects $X$ and $Y$, the set of morphisms $\mathbf{A}(X, Y)$ is an abelian group, and composition is bilinear. Furthermore, binary products and coproducts coincide, in the sense that the natural morphism $X \oplus Y \rightarrow X \times Y$ is an isomorphism.

We say that an object $X$ of an abelian category is indecomposable if whenever $X \cong U \oplus V$, either $U \cong 0$ or $V \cong 0$.

Example 2.8 Let Vec be the category of finite-dimensional vector spaces over some fixed field, $\mathbb{F}$. The morphisms are linear transformations. The zero object is (an element of the isomorphism class of) the trivial vector space, $0=\{0\}$. The product of $V$ and $W$ is the Cartesian (direct) product, $V \times W$. The coproduct is the direct sum, $V \oplus W$, which is canonically isomorphic to the direct product.

If $f: V \rightarrow W$ is linear, we set $\operatorname{ker} f=\{v \in V \mid f(v)=0\}, f(V)=\{f(v) \mid$ $v \in V\}$, and coker $f=W / f(V)$. It is a straightforward exercise to show that monomorphisms are simply injective linear transformations and that if $f$ is injective, then $V \cong f(V)$, and so $V$ is (isomorphic to) the kernel of the quotient map, $W \rightarrow$ coker $f$. Similarly, epimorphisms are surjective linear transformations, and by the First Homomorphism Theorem, if $f: V \rightarrow W$ is surjective, then $W$ is the cokernel of ker $f \rightarrow V$. Thus, Vec is an abelian category.

\subsection{Algebra}

We will need the following definitions in Lemma 4.5, which we use in the proof of Theorem 4.6.

A (nonnegatively) graded ring is a ring, $R$, along with a direct-sum decomposition, $R=\bigoplus_{n=0}^{\infty} R_{n}$, such that $1 \in R_{0}$, and if $a \in R_{m}$ and $b \in R_{n}$, then $a b \in R_{m+n}$. Our primary example will be the polynomial ring $\mathbb{F}[t]$ for a field $\mathbb{F}$ that is graded by degree.

A graded $\mathbb{F}[t]$-module is an $\mathbb{F}[t]$-module, $M$, with a decomposition $M=$ $\bigoplus_{n=0}^{\infty} M_{n}$ that satisfies $t^{m} x \in M_{m+n}$ whenever $x \in M_{n}$. We say that $M$ has finite type if each $M_{n}$ is finite dimensional over $\mathbb{F}$.

We will also make use of the following structure theorem for finitely generated modules over a principal ideal domain.

Theorem 2.9 [16, Theorem 6.12(ii), p. 225] Let A be a finitely generated module over a principal ideal domain $R$. Then $A$ is the direct sum of a free submodule $E$ of finite rank and a finite number of cyclic torsion modules. The cyclic torsion summands (if any) are of orders $p_{1}^{s_{1}}, \ldots, p_{k}^{s_{k}}$, where $p_{1}, \ldots, p_{k}$ are (not necessarily distinct) positive integers. The rank of $E$ and the list of ideals $\left(p_{1}^{s_{1}}\right), \ldots,\left(p_{k}^{s_{k}}\right)$ are uniquely determined by $A$ (except for the order of the $p_{i}$ ). 


\section{Interleavings of Diagrams}

In this section, we define $\varepsilon$-interleavings for $(\mathbb{R}, \leq)$-indexed diagrams and show that they induce a metric on a set of $(\mathbb{R}, \leq)$-indexed diagrams. Our definition is a categorical version of the definition in [5].

We consider the category $(\mathbb{R}, \leq)$ whose objects are the real numbers and the set of morphisms from $a$ to $b$ consists of a single morphism if $a \leq b$ and is otherwise empty. For $b \geq 0$, define $T_{b}:(\mathbb{R}, \leq) \rightarrow(\mathbb{R}, \leq)$ to be the functor given by $T_{b}(a)=a+b$, and define $\eta_{b}: \operatorname{Id}_{(\mathbb{R}, \leq)} \Rightarrow T_{b}$ to be the natural transformation given by $\eta_{b}(a): a \leq a+b$. Note that $T_{b} T_{c}=T_{b+c}$ and that $\eta_{b} \eta_{c}=\eta_{b+c}$.

Let $\mathbf{D}$ be any category, and let $\varepsilon \geq 0$. Let $F, G \in \mathbf{D}^{(\mathbb{R}, \leq)}$.

Definition 3.1 An $\varepsilon$-interleaving of $F$ and $G$ consists of natural transformations $\varphi: F \Rightarrow G T_{\varepsilon}$ and $\psi: G \Rightarrow F T_{\varepsilon}$, i.e.,

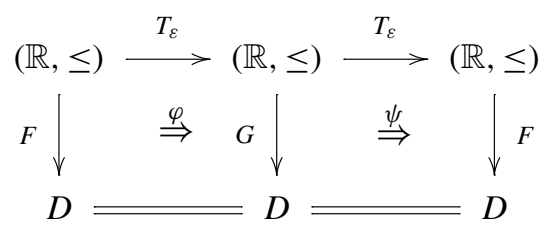

such that

$$
\left(\psi T_{\varepsilon}\right) \varphi=F \eta_{2 \varepsilon} \quad \text { and } \quad\left(\varphi T_{\varepsilon}\right) \psi=G \eta_{2 \varepsilon} .
$$

If $(F, G, \varphi, \psi)$ is an $\varepsilon$-interleaving, then we say that $F$ and $G$ are $\varepsilon$-interleaved.

The existence of the natural transformations $\varphi$ and $\psi$ implies that we have the following commutative diagrams for all $a \leq b$ :
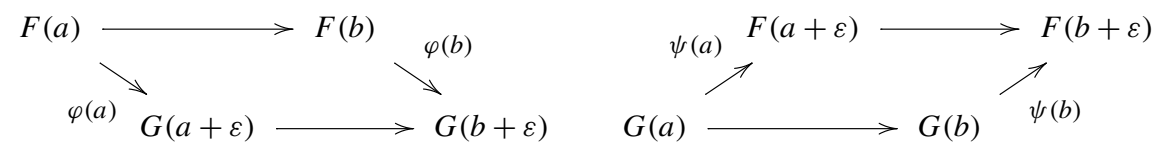

Identities (3) imply that the following diagrams commute for all $a$ :
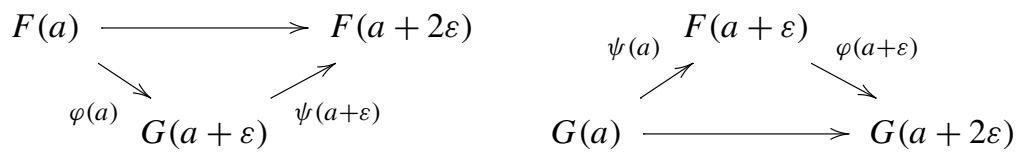

Definition 3.2 Say that $d(F, G) \leq \varepsilon$ if $F$ and $G$ are $\varepsilon$-interleaved. Explicitly,

$$
d(F, G)=\inf \{\varepsilon \geq 0 \mid F \text { and } G \text { are } \varepsilon \text {-interleaved }\},
$$

where we set $d(F, G)=\infty$ if $F$ and $G$ are not $\varepsilon$-interleaved for any $\varepsilon \geq 0$. 
We will show that this function $d$ is a generalized metric. It fails to be a metric because it can take the value $\infty$ and $d(F, G)=0$ does not imply that $F \cong G$. Notice that if $F$ and $G$ are 0 -interleaved, then $F \cong G$. However, $d(F, G)=0$ only implies that $F$ and $G$ are $\varepsilon$-interleaved for all $\varepsilon>0$. This does not imply that $F \cong G$. For an example, consider $F, G \in \operatorname{Vec}^{(\mathbb{R}, \leq)}$ where $F=0$ and $G(a)$ is the ground field for $a=0$ but is otherwise 0 . However, it does satisfy the other conditions of a metric, so it is an extended pseudometric.

Theorem 3.3 The function d defined above is an extended pseudometric on any subset of the class of $(\mathbb{R}, \leq)$-indexed diagrams in $\mathbf{D}$.

To prove the theorem, we will need the following lemma, which shows that the set of $\varepsilon$ for which two diagrams are $\varepsilon$-interleaved form a ray.

Lemma 3.4 If the $(\mathbb{R}, \leq)$-indexed diagrams $F$ and $G$ are $\varepsilon$-interleaved, then they are also $\varepsilon^{\prime}$-interleaved for any $\varepsilon^{\prime} \geq \varepsilon$.

Proof Let $\varphi: F \Rightarrow G T_{\varepsilon}$ and $\psi: G \Rightarrow F T_{\varepsilon}$ be such that $\left(\psi T_{\varepsilon}\right) \varphi=F \eta_{2 \varepsilon}$ and $\left(\varphi T_{\varepsilon}\right) \psi=G \eta_{2 \varepsilon}$.

Let $\varepsilon^{\prime} \geq \varepsilon$ and set $\bar{\varepsilon}=\varepsilon^{\prime}-\varepsilon$. Recall that we have the natural transformation, $\eta_{\bar{\varepsilon}}$ : $\operatorname{Id}_{(\mathbb{R}, \leq)} \Rightarrow T_{\bar{\varepsilon}}$, and thus, $\eta_{\bar{\varepsilon}} T_{\varepsilon}: T_{\varepsilon} \Rightarrow T_{\bar{\varepsilon}} T_{\varepsilon}=T_{\mathcal{E}^{\prime}}$. Therefore, $G \eta_{\bar{\varepsilon}} T_{\varepsilon}: G T_{\varepsilon} \Rightarrow G T_{\bar{\varepsilon}^{\prime}}$. Define $\hat{\varphi}=\left(G \eta_{\bar{\varepsilon}} T_{\varepsilon}\right) \varphi$. For example,

$$
\hat{\varphi}(a): F(a) \stackrel{\varphi(a)}{\longrightarrow} G(a+\varepsilon) \stackrel{G \eta_{\bar{\varepsilon}} T_{\varepsilon}(a)}{\longrightarrow} G\left(a+\varepsilon^{\prime}\right) .
$$

Similarly, define $\hat{\psi}=\left(F \eta_{\bar{\varepsilon}} T_{\varepsilon}\right) \psi$.

We see that $\left(\hat{\psi} T_{\mathcal{E}^{\prime}}\right) \hat{\varphi}=F \eta_{2 \varepsilon^{\prime}}$ from the following commutative diagram:

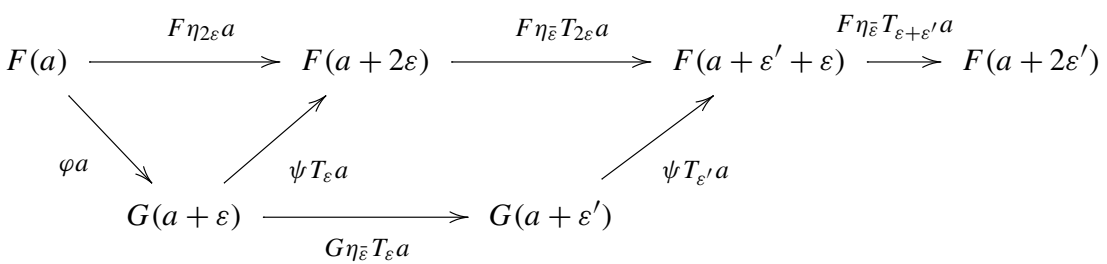

Similarly, one can check that $\left(\hat{\varphi} T_{\mathcal{E}^{\prime}}\right) \hat{\psi}=G \eta_{2 \varepsilon^{\prime}}$.

Proof of Theorem 3.3 The identity natural transformation shows that $d(F, F)=0$ for any diagram $F$. By the symmetry of the definition of $\varepsilon$-interleaving, we see that $d(F, G)=d(G, F)$ for any diagrams $F$ and $G$. It remains to show the triangle inequality.

Consider diagrams $F, G$, and $H$. Let $a=d(F, G)$ and $b=d(G, H)$. Let $\varepsilon>0$. Then by Lemma 3.4 and the definition of infimum, $F$ and $G$ are $(a+\varepsilon)$-interleaved, and $G$ and $H$ are $(b+\varepsilon)$-interleaved. Let $\varphi^{\prime}: F \Rightarrow G T_{a+\varepsilon}$ and $\psi^{\prime}: G \Rightarrow F T_{a+\varepsilon}$ and 
$\varphi^{\prime \prime}: G \Rightarrow H T_{b+\varepsilon}$ and $\psi^{\prime \prime}: H \Rightarrow G T_{b+\varepsilon}$ be the corresponding natural transformations. We will show that composing these natural transformations gives the desired natural transformations for an interleaving of $F$ and $H$.

Let $\varphi=\left(\varphi^{\prime \prime} T_{a+\varepsilon}\right) \varphi^{\prime}: F \Rightarrow H T_{b+\varepsilon} T_{a+\varepsilon}=H T_{a+b+2 \varepsilon}$ and $\psi=\left(\psi^{\prime} T_{b+\varepsilon}\right) \psi^{\prime \prime}$ : $H \Rightarrow F T_{a+\varepsilon} T_{b+\varepsilon}=F T_{a+b+2 \varepsilon}$. The first composition comes from the following diagram. The second is similar.

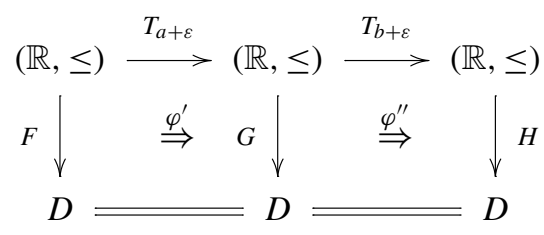

We claim that $\left(\psi T_{a+b+2 \varepsilon}\right) \varphi=F \eta_{2(a+b+2 \varepsilon)}$ and $\left(\varphi T_{a+b+2 \varepsilon}\right) \psi=H \eta_{2(a+b+2 \varepsilon)}$. The first identity comes from the following diagram. The second is similar.

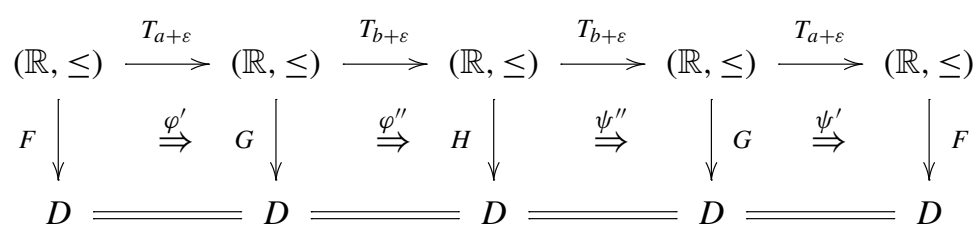

Thus, $F$ and $H$ are $(a+b+2 \varepsilon)$-interleaved for all $\varepsilon>0$. Therefore $d(F, H) \leq$ $a+b$.

Let us declare $F$ equivalent to $G$ if $d(F, G)=0$; this is an equivalence relation, and we obtain the following corollary.

Corollary 3.5 If we identify diagrams whose interleaving distance is 0 , then $d$ is an extended metric on this set of equivalence classes.

One of the mostly useful aspects of the categorical view of interleavings is that if we apply a functor to $\varepsilon$-interleaved diagrams, then the resulting diagrams are also $\varepsilon$-interleaved. That is,

Proposition 3.6 Let $F, G:(\mathbb{R}, \leq) \rightarrow \mathbf{D}$ and $H: \mathbf{D} \rightarrow \mathbf{E}$. If $F$ and $G$ are $\varepsilon$-interleaved, then so are $H F$ and $H G$. Thus,

$$
d(H F, H G) \leq d(F, G) .
$$

Proof Assume that $F$ and $G$ are $\varepsilon$-interleaved. Let $\varphi: F \Rightarrow G T_{\varepsilon}, \psi: G \Rightarrow F T_{\varepsilon}$ be the corresponding natural transformations. Then by functoriality, $H \varphi: H F \Rightarrow H G T_{\varepsilon}$ and $H \psi: H G \Rightarrow H F T_{\varepsilon}$, and $\left(H \psi T_{\varepsilon}\right)(H \varphi)=(H F) \eta_{2 \varepsilon}$ and $\left(H \varphi T_{\varepsilon}\right)(H \psi)=$ 
$(H G) \eta_{2 \varepsilon}$, as pictured in the following diagram:

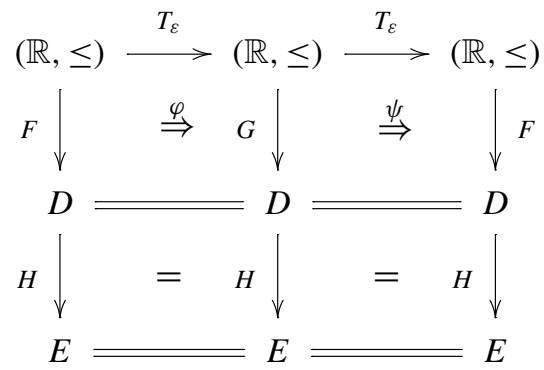

Therefore, $H F$ and $H G$ are $\varepsilon$-interleaved.

\section{Diagrams of Vector Spaces}

From our categorical point of view, persistent homology calculations are done on diagrams in the category Vec of finite-dimensional vector spaces over a fixed ground field $\mathbb{F}$. In this section, we study $(\mathbb{R}, \leq)$-indexed diagrams in Vec and define some of the usual characters in topological persistence in this setting: barcodes, persistence diagrams, and the bottleneck distance. Our main result is an isometric embedding of the set of finite barcodes with the bottleneck distance into the set of objects of $\operatorname{Vec}^{(\mathbb{R}, \leq)}$ with the interleaving distance.

The category Vec is one of the motivating examples of an abelian category. If the target category in a diagram category is an abelian category, then the diagram category inherits this structure. The necessary constructions are done objectwise. In

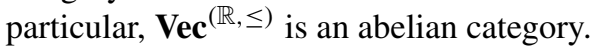

\subsection{Finite-Type Diagrams}

In this section we define finite-type and tame diagrams in Vec $\mathbf{V e}^{(\mathbb{R}, \leq)}$ and show that the two conditions are equivalent. As a corollary, we obtain a Krull-Schmidt theorem.

Definition 4.1 Given an interval $I \subseteq \mathbb{R}$, define the diagram $\chi_{I} \in \operatorname{Vec}^{(\mathbb{R}, \leq)}$ by

$$
\chi_{I}(a)=\left\{\begin{array}{ll}
\mathbb{F} & \text { if } a \in I, \\
0 & \text { otherwise },
\end{array} \quad \chi_{I}(a \leq b)= \begin{cases}\operatorname{Id}_{\mathbb{F}} & \text { if } a, b \in I \\
0 & \text { otherwise }\end{cases}\right.
$$

We say that a diagram $F \in \mathbf{V e c}^{(\mathbb{R}, \leq)}$ has finite type if $F \cong \bigoplus_{k=1}^{N} \chi_{I_{k}}$.

We remark that $\chi_{\mathbb{R}}$ and $\chi_{\emptyset}$ are the constant functors $\mathbb{F}$ and 0 , respectively.

Lemma 4.2 For an interval $I \subseteq \mathbb{R}$, the diagram $\chi_{I}$ is indecomposable. 
Proof Assume that $\chi_{I} \cong P \oplus Q$. If there is some $c \notin I$, then $P(c) \oplus Q(c) \cong$ $\chi_{I}(c)=0$, and therefore $P(c)=Q(c)=0$.

Let $a \in I$. Then $P(a) \oplus Q(a) \cong \chi_{I}(a) \cong \mathbb{F}$. Without loss of generality, assume that $P(a) \cong \mathbb{F}$ and $Q(a)=0$. Let $a \leq b \in I$. Since $Q(a)=0, Q(a \leq b)=0$. Thus, it follows from $P(a \leq b) \oplus Q(a \leq b)=(P \oplus Q)(a \leq b) \cong \chi_{I}(a \leq b)=\operatorname{Id}_{\mathbb{F}}$ that $P(a \leq b) \cong \operatorname{Id}_{\mathbb{F}}$. Hence, from $P(b) \oplus Q(b) \cong \chi_{I}(b) \cong \mathbb{F}$ we get that $P(b) \cong \mathbb{F}$ and $Q(b)=0$. Similarly, if $d \leq a \in I$, we get that $Q(d \leq a)=0, P(d \leq a) \cong \operatorname{Id}_{\mathbb{F}}$, $P(d) \cong \mathbb{F}$ and $Q(d)=0$.

We have shown that $P \cong \chi_{I}$ and $Q=0$. Therefore, $\chi_{I}$ is indecomposable.

The following definitions are variations of those in [7].

Definition 4.3 Let $F \in \operatorname{Vec}^{(\mathbb{R}, \leq)}$. Let $I \subseteq \mathbb{R}$ be an interval. Say that $F$ is constant on $I$ if for all $a \leq b \in I, F(a \leq b)$ is an isomorphism. We call $a \in \mathbb{R}$ a regular value of $F$ if there is some open interval $I \ni a$ such that $F$ is constant on $I$. Otherwise, we call $a$ a critical value of $F{ }^{1}$ We call $F$ tame if it has a finite number of critical values.

Lemma 4.4 (Critical Value Lemma) If an interval I does not contain any critical values of $F$, then $F$ is constant on $I$.

Proof Let $a \leq b \in I$. By assumption, for all $c \in[a, b]$, there exists an interval $I_{c} \ni c$ such that $F$ is constant on $I_{c}$. Since $[a, b]$ is compact, the cover $\left\{I_{c} \mid c \in[a, b]\right\}$ has a finite subcover $\left\{I_{c_{1}}, \ldots, I_{c_{n}}\right\}$. Choose a sequence $a=d_{0} \leq d_{1} \leq \cdots \leq d_{m+1}=b$ such that for all $0 \leq k \leq m, d_{k}, d_{k+1} \in I_{c_{j}}$ for some $1 \leq j \leq n$. Then $F\left(d_{k} \leq d_{k+1}\right)$ is an isomorphism for $0 \leq k \leq m$, and thus $F(a \leq b)$ is an isomorphism.

We will need the following lemma in the proof of Theorem 4.6. We refer the reader to Sect. 2.4 for the definition of a finite-type graded $\mathbb{F}[t]$-module.

Lemma 4.5 The category $\operatorname{Vec}^{(\mathbb{Z}, \leq)}$ is isomorphic to the category of finite-type graded $\mathbb{F}[t]$-modules.

Proof To each diagram $F \in \mathbf{V e c}^{(\mathbb{Z}, \leq)}$, we can assign the finite-type graded $\mathbb{F}[t]$ module $M$, where for $k \in \mathbb{Z}, M_{k}=F(k)$, and for $a \in M_{k}, t \cdot a=F(k \leq k+1)(a)$.

To each finite-type graded $\mathbb{F}[t]$-module $M$, we can assign the diagram $F \in$ $\operatorname{Vec}^{(\mathbb{Z}, \leq)}$ given by $F(k)=M_{k}$ and whose morphisms are generated by $F(k \leq$ $k+1)(a)=t \cdot a$ for $a \in F(k)$.

Both composites of these two functors are equal to the identity functor.

Theorem 4.6 A diagram in $\operatorname{Vec}^{(\mathbb{R}, \leq)}$ is tame if and only if it has finite type.

\footnotetext{
${ }^{1}$ Even if $F$ is induced by sublevel sets, it is inadequate to define $a \in \mathbb{R}$ to be a critical value of $F$ if for all sufficiently small $\varepsilon>0$, the map $F(a-\varepsilon \leq a+\varepsilon)$ is not an isomorphism [7]. Consider the example $X=$ $\left\{(x, y) \in \mathbb{R}^{2} \mid 0 \leq x \leq 1,0<y \leq 1\right\}$ and $f(x, y)$ equal to 0 if $x=0,-1$ if $x=1$, and $y$ otherwise. Then 0 is not a critical value under this stricter definition, but the map $H_{0}\left(f^{-1}(-\infty, 0]\right) \rightarrow H_{0}\left(f^{-1}(-\infty, 1]\right)$ induced by inclusion is not an isomorphism, contradicting the Critical Value Lemma.
} 
Proof To prove the "if" statement, we consider an interval $I \subseteq \mathbb{R}$. By definition, $a \in \mathbb{R}$ is a critical value of $\chi_{I}$ if and only if $a$ is an endpoint of $\bar{I}$. Let $F \in \operatorname{Vec}^{(\mathbb{R}, \leq)}$ such that $F \cong \bigoplus_{k=1}^{N} \chi_{I_{k}}$. Then $a \in \mathbb{R}$ is a critical value of $F$ if and only if it is an endpoint of one of the intervals $I_{k}$, and so $F$ is tame.

The remainder of the proof is devoted to establishing the "only if" statement. Assume that $F \in \operatorname{Vec}^{(\mathbb{R}, \leq)}$ has critical values $a_{1}<a_{2}<\cdots<a_{n}$. Choose $b_{0}, \ldots, b_{n}$ such that $b_{0}<a_{1}$ for $k \in\{1, \ldots, n-1\}, a_{k}<b_{k}<a_{k+1}$, and $a_{n}<b_{n}$. For convenience, set $a_{0}=-\infty, a_{n+1}=\infty$ and $F\left(a_{0}\right)=F\left(b_{0}\right), F\left(a_{n+1}\right)=F\left(b_{n}\right)$. We have the ordered sequence

$$
-\infty=a_{0}<b_{0}<a_{1}<b_{1}<a_{2}<\cdots<b_{n-1}<a_{n}<b_{n}<a_{n+1}=\infty .
$$

We now identify the finite-valued part of this sequence with the integers from 0 to $2 n$.

More precisely, we define a functor $i:[2 n] \rightarrow(\mathbb{R}, \leq)$ given by

$$
k \mapsto \begin{cases}b_{\frac{k}{2}} & \text { if } k \text { is even }, \\ a_{\frac{k+1}{2}} & \text { if } k \text { is odd. }\end{cases}
$$

We also define a functor $r:(\mathbb{R}, \leq) \rightarrow[2 n]$ given by

$$
c \mapsto \begin{cases}2 k-1 & \text { if } c=a_{k}, k \in\{1, \ldots, n\}, \\ 2 k & \text { if } a_{k}<c<a_{k+1}, k \in\{0, \ldots, n\} .\end{cases}
$$

Then we have the composite functor ir $:(\mathbb{R}, \leq) \rightarrow(\mathbb{R}, \leq)$ given by

$$
c \mapsto \begin{cases}a_{k} & \text { if } c=a_{k}, k \in\{1, \ldots, n\}, \\ b_{k} & \text { if } a_{k}<c<a_{k+1}, k \in\{0, \ldots, n\} .\end{cases}
$$

Precomposing $F$ with this functor gives us an induced functor $(i r)^{*} F \in \operatorname{Vec}^{(\mathbb{R}, \leq)}$. That is, $(i r)^{*} F(c)=F(i r c) .^{2}$ Notice that by Lemma $4.4, F(i r c) \cong F(c)$ for all $c \in \mathbb{R}$. Thus, $(i r)^{*}: F \mapsto(i r)^{*} F$ is a natural isomorphism.

Next, $i^{*} F:[2 n] \rightarrow$ Vec can be extended to a functor $i^{*} F:(\mathbb{Z}, \leq) \rightarrow$ Vec by setting $\left(i^{*} F\right)(k)=\left(i^{*} F\right)(0)$ for $k<0$, with $i^{*} F(k \leq 0)$ the identity, and for $k>2 n$, setting $\left(i^{*} F\right)(k)=\left(i^{*} F\right)(2 n)$ with $i^{*} F(2 n \leq k)$ the identity. By Lemma 4.5, we can consider $i^{*} F$ to be a graded $\mathbb{F}[t]$-module. Note that by assumption, $i^{*} F$ is a finitely generated graded $\mathbb{F}[t]$-module.

By the structure theorem for finitely generated graded modules over a principal ideal domain (Theorem 2.9), there is a unique decomposition,

$$
i^{*} F \cong\left(\bigoplus_{i=1}^{n_{1}} t^{c_{i}} \mathbb{F}[t]\right) \oplus\left(\bigoplus_{j=1}^{n_{2}} t^{d_{j}}\left(\mathbb{F}[t] /\left(t^{e_{j}}\right)\right)\right) .
$$

\footnotetext{
${ }^{2}$ Our notation comes from category theory; an arrow $f: x \rightarrow y$ defines a natural map $f^{*}: \operatorname{Hom}(y, z) \rightarrow$ $\operatorname{Hom}(x, z)$ obtained by precomposing a given arrow $y \rightarrow z$ with $f$.
} 
It follows that as elements of $\operatorname{Vec}^{(\mathbb{Z}, \leq)}$,

$$
i^{*} F \cong\left(\bigoplus_{i=1}^{n_{1}} \chi_{\left[c_{i}, \infty\right)}\right) \oplus\left(\bigoplus_{j=1}^{n_{2}} \chi_{\left[d_{j}, d_{j}+e_{j}\right)}\right)
$$

Therefore,

$$
F \cong(i r)^{*} F=r^{*} i^{*} F \cong\left(\bigoplus_{i=1}^{n_{1}} r^{*} \chi_{\left[c_{i}, \infty\right)}\right) \oplus\left(\bigoplus_{j=1}^{n_{2}} r^{*} \chi_{\left[d_{j}, d_{j}+e_{j}\right)}\right),
$$

where

$$
r^{*} \chi_{[k, \infty)}= \begin{cases}\chi_{\left[a_{\frac{k+1}{2}}, \infty\right)} & \text { if } k \text { odd } \\ \chi_{\left(a_{\frac{k}{2}}, \infty\right)} & \text { if } k \text { even }\end{cases}
$$

and

$$
r^{*} \chi_{[k, \ell)}= \begin{cases}\chi_{\left[a_{\frac{k+1}{2}}, a_{\frac{\ell+1}{2}}\right)} & \text { if } k, \ell \text { odd, } \\ \chi_{\left[a_{\frac{k+1}{2}}, a_{\frac{\ell}{2}}\right]} & \text { if } k \text { odd, } \ell \text { even, } \\ \chi_{\left(a_{\frac{k}{2}}, a_{\frac{\ell+1}{2}}\right)} & \text { if } k \text { even, } \ell \text { odd, } \\ \chi_{\left(a_{\frac{k}{2}}, a_{\frac{\ell+1}{2}}\right]} & \text { if } k, l \text { even. }\end{cases}
$$

Thus, $F$ has finite type.

By the uniqueness of the decomposition in the structure theorem for graded modules over a graded PID in the previous proof, we get that finite-type diagrams in $\operatorname{Vec}^{(\mathbb{R}, \leq)}$ satisfy the following Krull-Schmidt theorem. Compare this with [4, Proposition 2.2].

Corollary 4.7 (Krull-Schmidt) If $F \cong \bigoplus_{k=1}^{n} \chi_{I_{k}}$ and $F \cong \bigoplus_{j=1}^{m} \chi_{I_{j}^{\prime}}$, then $n=m$ and the sequences $I_{1}, \ldots, I_{n}$ and $I_{1}^{\prime}, \ldots, I_{m}^{\prime}$ are the same up to reordering.

\subsection{Barcodes and Persistence Diagrams}

Here we define barcodes and persistence diagrams for finite-type diagrams in $\operatorname{Vec}^{(\mathbb{R}, \leq)}$. We observe that finite-type diagrams in $\mathbf{V e c}^{(\mathbb{R}, \leq)}$ are a categorification of finite barcodes.

Definition 4.8 Assume that $F \in \mathbf{V e c}^{(\mathbb{R}, \leq)}$ has finite type. A barcode is a multiset of intervals. The barcode of $F$ is the multiset $\left\{I_{k}\right\}_{k=1}^{n}$ where $F \cong \bigoplus_{k=1}^{n} \chi_{I_{k}}$. This is well defined by Corollary 4.7, which follows from Theorem 4.6.

A persistence diagram is a multiset of increasing pairs of extended real numbers. The persistence diagram of $F$ is the multiset $\left\{\left(a_{k}, b_{k}\right)\right\}_{k=1}^{n}$, where $a_{k} \leq b_{k}$ and $\left\{a_{k}, b_{k}\right\}$ are the endpoints of $I_{k}$, with $F \cong \bigoplus_{k=1}^{n} \chi_{I_{k}}$. Again, this is well defined by Corollary 4.7 . 
By Corollary 4.7, we immediately have the following. Compare with [21, Corollary 3.1] and [12, Persistence Equivalence Theorem]. Note that a finite barcode is a finite multiset of intervals, not a multiset of finite intervals.

Corollary 4.9 (Categorification of Barcodes) There is a bijection between isomorphism classes of finite-type diagrams in $\mathbf{V e c}^{(\mathbb{R}, \leq)}$ and finite barcodes.

\subsection{Bottleneck Distance}

In this section, we define the bottleneck distance between two barcodes in terms of the interleaving distance. We show that this results in the usual definition of [7]. We end by proving an isometric embedding of the set of finite barcodes with the bottleneck distance into the set of $(\mathbb{R}, \leq)$-indexed diagrams in Vec with the interleaving distance.

Definition 4.10 Given multisets $A$ and $B$, define the multiset $A_{B}$ to be the disjoint union of $A$ and the multiset containing the empty interval $\emptyset$ with cardinality $|B|$. A stable bijection or partial matching between two multisets $A$ and $B$ is a bijection, $f: A_{B} \rightarrow B_{A}$. Write $f: A \rightleftharpoons B$.

Definition 4.11 Let $B$ and $B^{\prime}$ be two barcodes. Define the bottleneck distance between $B$ and $B^{\prime}$ by

$$
d_{B}\left(B, B^{\prime}\right)=\inf _{f: B \rightleftharpoons B^{\prime}} \sup _{I \in \operatorname{dom} f} d\left(\chi_{I}, \chi_{f(I)}\right) .
$$

On the right-hand side of (4) we have the interleaving distance. It follows from the following two propositions that this definition of bottleneck distance is equivalent to that in [7].

Proposition 4.12 Let $I$ and $I^{\prime}$ be two finite intervals.

(1) If $I=I^{\prime}=\emptyset$, then $d\left(\chi_{I}, \chi_{I^{\prime}}\right)=0$.

(2) If $I^{\prime}=\emptyset$ and $I$ has endpoints $a$ and $b$, then $d\left(\chi_{I}, \chi_{I^{\prime}}\right)=\frac{b-a}{2}$.

(3) If I and $I^{\prime}$ have endpoints $a, b$ and $a^{\prime}, b^{\prime}$, respectively, then

$$
d\left(\chi_{I}, \chi_{I^{\prime}}\right)=\min \left(\max \left(\left|a-a^{\prime}\right|,\left|b-b^{\prime}\right|\right), \max \left(\frac{b-a}{2}, \frac{b^{\prime}-a^{\prime}}{2}\right)\right) .
$$

Proposition 4.13 Let $I$ and $I^{\prime}$ be two intervals, at least one of which is infinite.

(1) If $I=I^{\prime}=\mathbb{R}$, then $d\left(\chi_{I}, \chi_{I^{\prime}}\right)=0$.

(2) If $\inf (I)=\inf \left(I^{\prime}\right)=-\infty$ and $I$ and $I^{\prime}$ have right endpoints $b$ and $b^{\prime}$, then $d\left(\chi_{I}, \chi_{I^{\prime}}\right)=\left|b-b^{\prime}\right|$.

(3) If $\sup (I)=\sup \left(I^{\prime}\right)=\infty$ and $I$ and $I^{\prime}$ have left endpoints $a$ and $a^{\prime}$, then $d\left(\chi_{I}, \chi_{I^{\prime}}\right)=\left|a-a^{\prime}\right|$.

(4) In all other cases, $d\left(\chi_{I}, \chi_{I^{\prime}}\right)=\infty$. 
Propositions 4.12 and 4.13 follow from the following two lemmas. The proofs are technical yet straightforward, and we leave them to the motivated reader.

Assume that the intervals $I$ and $I^{\prime}$ are finite. Let $h$ and $h^{\prime}$ each denote half the length of the interval $I$ and $I^{\prime}$, respectively, where the length of $\emptyset$ is 0 . If $I$ and $I^{\prime}$ are nonempty, let $m$ and $m^{\prime}$ denote their respective midpoints.

Lemma 4.14 Assume that $I$ and $I^{\prime}$ are finite intervals. $d\left(\chi_{I}, \chi_{I^{\prime}}\right) \leq \max \left(h, h^{\prime}\right)$.

Proof Let $\varepsilon>\max \left(h, h^{\prime}\right)$. Then $\chi_{I} \eta_{2 \varepsilon}=0=\chi_{I^{\prime}} \eta_{2 \varepsilon}$. Let $\varphi=0$ and $\psi=0$. Then $\varphi$ and $\psi$ give an $\varepsilon$-interleaving of $\chi_{I}$ and $\chi_{I^{\prime}}$.

Lemma 4.15 Assume that $I$ and $I^{\prime}$ are finite intervals. If $m \notin I^{\prime}$, then $d\left(\chi_{I}, \chi_{I^{\prime}}\right) \geq h$.

Proof Let $\varepsilon<h$. Then $[m-\varepsilon, m+\varepsilon] \subset I$. Thus, $\chi_{I} \eta_{2 \varepsilon}(m-\varepsilon)=\operatorname{Id}_{\mathbb{F}}$. Suppose $m \notin I^{\prime}$. Assume that there exists an $\varepsilon$-interleaving $(\varphi, \psi)$ of $\chi_{I}$ and $\chi_{I^{\prime}}$. Then $\left(\psi T_{\varepsilon}\right) \varphi(m-\varepsilon)=\operatorname{Id}_{\mathbb{F}}$. But $\varphi(m-\varepsilon) \in \chi_{I^{\prime}}(m)=0$. Therefore, $\left(\psi T_{\varepsilon}\right) \varphi(m-\varepsilon)=0$, which is a contradiction. Thus, $d\left(\chi_{I}, \chi_{I^{\prime}}\right) \geq h$.

In the statement of the following theorem, we abuse notation slightly by using $\operatorname{Vec}^{(\mathbb{R}, \leq)}$ to denote the set of objects in the category $\operatorname{Vec}^{(\mathbb{R}, \leq)}$.

Theorem 4.16 (Categorification of the Metric Space of Persistence Diagrams) Let $\mathcal{B}$ be the set of finite barcodes, $d_{B}$ the bottleneck distance, and $d$ the interleaving distance. The mapping $\chi$ defined by $\chi\left(\left\{I_{k}\right\}_{k=1}^{n}\right)=\bigoplus_{k=1}^{n} \chi_{I_{k}}$ gives an isometric embedding of metric spaces

$$
\chi:\left(\mathcal{B}, d_{B}\right) \hookrightarrow\left(\operatorname{Vec}^{(\mathbb{R}, \leq)}, d\right)
$$

Proof Let $B, B^{\prime} \in \mathcal{B}$. By [5, Theorem 4.4], we know that $d_{B}\left(B, B^{\prime}\right) \leq d(\chi(B)$, $\left.\chi\left(B^{\prime}\right)\right)$. It remains to show that

$$
d\left(\chi(B), \chi\left(B^{\prime}\right)\right) \leq d_{B}\left(B, B^{\prime}\right) .
$$

If $d_{B}\left(B, B^{\prime}\right)=\infty$, then this is trivial. Assume that $d_{B}\left(B, B^{\prime}\right)<\infty$.

Let $f: B \rightleftharpoons B^{\prime}$ such that $\sup _{I \in \operatorname{dom}(f)} d\left(\chi_{I}, \chi_{f(I)}\right)<\infty$. Choose $\varepsilon>$ $\sup _{I \in \operatorname{dom}(f)} d\left(\chi_{I}, \chi_{f(I)}\right)$. By Lemma 3.4, for each $I \in \operatorname{dom}(f), \chi_{I}$ and $\chi_{f(I)}$ are $\varepsilon$-interleaved. By Corollary 7.11, $\chi(B)$ and $\chi\left(B^{\prime}\right)$ are $\varepsilon$-interleaved.

Thus, $\chi(B)$ and $\chi\left(B^{\prime}\right)$ are $\varepsilon$-interleaved for all $\varepsilon>d_{B}\left(B, B^{\prime}\right)$. It follows that $d\left(\chi(B), \chi\left(B^{\prime}\right)\right) \leq d_{B}\left(B, B^{\prime}\right)$.

\section{Stability}

In [7], Cohen-Steiner, Edelsbrunner, and Harer prove that persistent homology of sublevel sets of a function is stable with respect to perturbations of the function as measured by the supremum norm. In this section, we use our categorical framework to generalize this Stability Theorem, as well as its generalization in [5]. 
Let $X \in$ Top. Assume that $f, g: X \rightarrow \mathbb{R}$. Note that we do not require that $f$ and $g$ be continuous. Let $F \in \operatorname{Top}^{(\mathbb{R}, \leq)}$ be defined by $F(a)=f^{-1}(\infty, a]$ for $a \in \mathbb{R}$ and $F(a \leq b)$ is given by inclusion. Define $G$ similarly using $g$. Let $H: \mathbf{T o p} \rightarrow \mathbf{D}$ be any functor, e.g., singular homology with coefficients in a field $\mathbb{F}$, or rational homotopy groups. Recall that $\|f-g\|_{\infty}=\sup _{x \in X}|f(x)-g(x)|$.

Theorem 5.1 (Stability Theorem)

$$
d(H F, H G) \leq\|f-g\|_{\infty} .
$$

Proof Let $\varepsilon=\|f-g\|_{\infty}$. First, we observe that by the assumption,

$$
F(a)=f^{-1}(-\infty, a] \subseteq g^{-1}(-\infty, a+\varepsilon]=G(a+\varepsilon),
$$

and similarly, $G(a) \subseteq F(a+\varepsilon)$. Thus, $F$ and $G$ are $\varepsilon$-interleaved. It follows that $H F$ and $H G$ are $\varepsilon$-interleaved (Proposition 3.6), and thus

$$
d(H F, H G) \leq\|f-g\|_{\infty} .
$$

\section{Extended Persistence}

In [8], Cohen-Steiner, Edelsbrunner, and Harer, define extended persistence to obtain a sequence of vector spaces in which the homology classes of the total space do not live forever. Given a simplicial complex $K$ on $n$ ordered vertices, let $K_{i}$ be the subcomplex spanned by the first $i$ vertices, and let $L_{i}$ be the subcomplex spanned by the last $i$ vertices. Let $H_{k}$ denote degree $k$ relative simplicial homology with coefficients in the field $\mathbb{Z} / 2 \mathbb{Z}$. Then they construct the sequence

$$
\begin{aligned}
0 & =H_{k}\left(K_{0}, \emptyset\right) \rightarrow H_{k}\left(K_{1}, \emptyset\right) \rightarrow \cdots \rightarrow H_{k}\left(K_{n}, \emptyset\right) \\
& =H_{k}\left(K, L_{0}\right) \rightarrow H_{k}\left(K, L_{1}\right) \rightarrow \cdots \rightarrow H_{k}\left(K, L_{n}\right)=0 .
\end{aligned}
$$

They show that the Stability Theorem of [7] can be applied in this case. Here we give a generalization of this construction and the corresponding stability theorem.

Let $X \in$ Top. Assume that $f: X \rightarrow \mathbb{R}$, where $f$ need not be continuous, and there exists an $M \in \mathbb{R}$ such that $f(x) \leq M$ for all $x \in X$. Let $s>0$ be an arbitrary amount to space out the upward and downward filtrations. Define the $(\mathbb{R}, \leq)$-indexed diagram of pairs of topological spaces, $F \in$ Pair $^{(\mathbb{R}, \leq)}$, as follows.

For $c<M+s$, let $F(c)=\left(f^{-1}(-\infty, c], \emptyset\right)$. For $c \geq M+s$, let $F(c)=$ $\left(X, f^{-1}[2 M+s-c, \infty)\right)$. Notice that for $M \leq c<M+s, F(c)=(X, \emptyset)$, and $F(M+s)=\left(X, f^{-1}(M)\right)$.

For $c \leq d, F(c \leq d)$ is given by inclusion. Indeed, if $c \leq d<M+s$, we have $\left(f^{-1}(-\infty, c], \emptyset\right) \subseteq\left(f^{-1}(-\infty, d], \emptyset\right)$, if $M+s \leq c \leq d$, then $\left(X, f^{-1}[2 M+s-\right.$ $c, \infty) \subseteq\left(X, f^{-1}[2 M+s-d, \infty)\right)$, and if $c<M+s \leq d$, then $\left(f^{-1}(-\infty, c], \emptyset\right) \subseteq$ $\left(X, f^{-1}[2 M+s-d, \infty)\right)$.

In the special case that there exists an $m \in \mathbb{R}$ such that $f(x) \geq m$ for all $x \in X$, then for $c<m, F(c)=(\emptyset, \emptyset)$, and for $c \geq M+s+(M-m)=2 M+s-m, F(c)=$ $(X, X)$. 
Now assume that we also have another (not necessarily continuous) map $g: X \rightarrow$ $(-\infty, M]$. Define $G \in$ Pair $^{(\mathbb{R}, \leq)}$ similarly. Let $H:$ Pair $\rightarrow \mathbf{D}$ be any functor, e.g., relative homology with coefficients in some field $\mathbb{F}$.

Theorem 6.1 (Stability Theorem for Extended Persistence)

$$
d(H F, H G) \leq\|f-g\|_{\infty} .
$$

Proof Let $\varepsilon=\|f-g\|_{\infty}$. Let $c \in \mathbb{R}$. Then by assumption, $\left(f^{-1}(-\infty, c], \emptyset\right) \subseteq$ $\left(g^{-1}(-\infty, c+\varepsilon], \emptyset\right),\left(X, f^{-1}[2 M+s-c, \infty)\right) \subseteq\left(X, g^{-1}[2 M+s-(c+\varepsilon), \infty)\right)$, and $\left(f^{-1}(-\infty, c], \emptyset\right) \subseteq\left(X, g^{-1}[2 M+s-(c+\varepsilon), \infty)\right)$. Also, by assumption, we have the same relations with $f$ and $g$ switched. Thus, $F$ and $G$ are $\varepsilon$-interleaved. It follows that $H F$ and $H G$ are $\varepsilon$-interleaved (Proposition 3.6), and thus

$$
d(H F, H G) \leq d(F, G) \leq\|f-g\|_{\infty} .
$$

\section{Abelian Structure of Interleavings}

Let $\mathbf{D}$ be a category, and let $\varepsilon \geq 0$. In this section, we consider the category $\operatorname{Int}_{\varepsilon}(\mathbf{D})$ of $\varepsilon$-interleavings of diagrams in $\mathbf{D}^{(\mathbb{R}, \leq)}$, which we define below. We will show that this construction is functorial and that if $\mathbf{D}$ is an abelian category, then so is $\operatorname{Int}_{\varepsilon}(\mathbf{D})$. As a corollary, we obtain stability theorems for kernels, images, and cokernels in persistence and extended persistence.

First, let us recall that the functor $T_{\varepsilon}: \mathbb{R} \rightarrow \mathbb{R}, T_{\varepsilon}(x)=x+\varepsilon, T_{\varepsilon}(x \leq y)=x+$ $\varepsilon \leq y+\varepsilon$, comes equipped with a "unit" natural transformation, $\eta_{\varepsilon}: \operatorname{Id} \Rightarrow T_{\varepsilon}$, since $x \leq x+\varepsilon$. We will write $\eta_{\varepsilon}^{2}$ for the iteration $\mathrm{Id} \Rightarrow T_{\varepsilon} \Rightarrow T_{\varepsilon}^{2}$.

Definition 7.1 The objects of $\operatorname{Int}_{\varepsilon}(\mathbf{D})$ are $\varepsilon$-interleavings, $(F, G, \varphi, \psi)$, where $\varphi$ : $F \Rightarrow G T_{\varepsilon}, \psi: G \Rightarrow F T_{\varepsilon}$, such that $\left(\psi T_{\varepsilon}\right) \varphi=F \eta_{\varepsilon}^{2}$ and $\left(\varphi T_{\varepsilon}\right) \psi=G \eta_{\varepsilon}^{2}$ (Definition 3.1). A morphism $(\alpha, \beta):(F, G, \varphi, \psi) \Rightarrow\left(F^{\prime}, G^{\prime}, \varphi^{\prime}, \psi^{\prime}\right)$ consists of a pair of natural transformations, $\alpha: F \Rightarrow F^{\prime}$ and $\beta: G \rightarrow G^{\prime}$, such that the diagrams

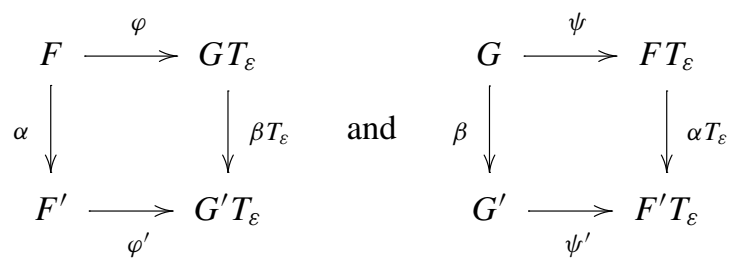

commute.

Let us also verify the naturality of the above construction.

Proposition 7.2 Definition 7.1 of $\operatorname{Int}_{\varepsilon}(\mathbf{D})$ is functorial in $\varepsilon$ and in $\mathbf{D}$. 
Proof Let $\varepsilon \leq \varepsilon^{\prime}$. The functor, $\operatorname{Int}_{\varepsilon}(\mathbf{D}) \rightarrow \mathbf{I n t}_{\varepsilon^{\prime}}(\mathbf{D})$, is defined on objects by Lemma 3.4. To be precise, we have $(F, G, \varphi, \psi) \mapsto(F, G, \hat{\varphi}, \hat{\psi})$, where $\hat{\varphi}=$ $\left(G \eta_{\varepsilon^{\prime}-\varepsilon} T_{\varepsilon}\right) \varphi$, and $\hat{\psi}=\left(F \eta_{\varepsilon^{\prime}-\varepsilon} T_{\varepsilon}\right) \psi$. Let $(\alpha, \beta):(F, G, \varphi, \psi) \rightarrow\left(F^{\prime}, G^{\prime}, \varphi^{\prime}, \psi^{\prime}\right) \in$ $\operatorname{Int}_{\varepsilon}(\mathbf{D})$. Then the commutative diagram

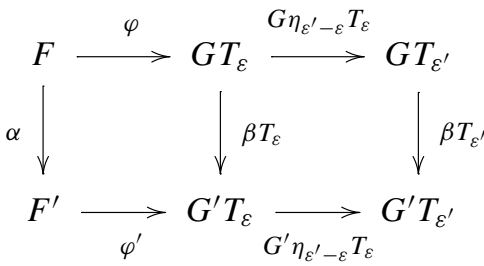

and a similar one show that $(\alpha, \beta):(F, G, \hat{\varphi}, \hat{\psi}) \rightarrow\left(F^{\prime}, G^{\prime}, \hat{\varphi}^{\prime}, \hat{\psi}^{\prime}\right) \in \mathbf{I n t}_{\varepsilon^{\prime}}(\mathbf{D})$. From this it follows that $\operatorname{Int}_{\varepsilon}(\mathbf{D})$ is functorial in $\varepsilon$.

Now consider a functor $H: \mathbf{D} \rightarrow \mathbf{D}^{\prime}$. The induced functor $\operatorname{Int}_{\varepsilon}(\mathbf{D}) \rightarrow \operatorname{Int}_{\varepsilon}\left(\mathbf{D}^{\prime}\right)$ is defined by composition with $H$ (for the details of the definition on objects, see the proof of Proposition 3.6). It follows that $\mathbf{I n t}_{\varepsilon}(\mathbf{D})$ is functorial in D.

Let $\mathbf{A}$ be an abelian category. Then, as discussed at the start of Sect. 4 , so is $\mathbf{A}^{(\mathbb{R}, \leq)}$. We claim that $\operatorname{Int}_{\varepsilon}(\mathbf{A})$ is also an abelian category. Recall (Sect. 2.3.4) that a category is abelian if it has a zero object, all finite products and coproducts, every morphism has a kernel and a cokernel, and all monomorphisms and epimorphisms are kernels and cokernels, respectively.

Lemma 7.3 The category $\operatorname{Int}_{\varepsilon}(\mathbf{A})$ has a zero object.

Proof The zero object of $\mathbf{I n t}_{\varepsilon}(\mathbf{A})$ comes from the zero object, 0, of $\mathbf{A}$. The diagram category $\operatorname{Vec}^{(\mathbb{R}, \leq)}$ then inherits the constant zero diagram, $O_{x}=0$ for all $x \in \mathbb{R}$, with the identity morphism $O_{x} \rightarrow O_{y}$ for $x \leq y$. In turn, we define the trivial $\varepsilon$ interleaving, $(O, O, \omega, \omega)$, where $\omega_{x}: O_{x} \rightarrow O_{x+\varepsilon}$ is again the identity. It is easy to see that $(O, O, \omega, \omega)$ is the desired zero object. Indeed, to see that it is initial, we note that for every interleaving $(F, G, \varphi, \psi)$ and for every $x \in \mathbb{R}$, there are unique morphisms $O_{x} \rightarrow F_{x}$ and $O_{x} \rightarrow G_{x}$ because $O_{x}$ is initial in $\mathbf{A}$, and the appropriate diagrams commute. Similarly, $(O, O, \omega, \omega)$ is final, and hence the desired zero object in $\operatorname{Int}_{\varepsilon}(\mathbf{A})$.

In particular, for any objects $X, Y \in \operatorname{Int}_{\varepsilon}(\mathbf{A})$, we now have the zero morphism $0: X \rightarrow Y$, that is, the composite $X \rightarrow O \rightarrow Y$.

Lemma 7.4 The category $\mathbf{I n t}_{\varepsilon}(\mathbf{A})$ has all pull-backs and push-outs, and their components in $\mathbf{A}^{(\mathbb{R}, \leq)}$ are given by the respective pull-backs and push-outs in $\mathbf{A}^{(\mathbb{R}, \leq)}$.

Proof We show that $\mathbf{I n t}_{\varepsilon}(\mathbf{A})$ has all pull-backs. The arguments and constructions for push-outs are dual. 
Consider the diagram

$$
\left(F^{\prime}, G^{\prime}, \varphi^{\prime}, \psi^{\prime}\right) \stackrel{\left(\alpha^{\prime}, \beta^{\prime}\right)}{\longrightarrow}(F, G, \varphi, \psi) \stackrel{\left(\alpha^{\prime \prime}, \beta^{\prime \prime}\right)}{\longleftarrow}\left(F^{\prime \prime}, G^{\prime \prime}, \varphi^{\prime \prime}, \psi^{\prime \prime}\right) .
$$

The category $\mathbf{A}^{(\mathbb{R}, \leq)}$ is abelian. Thus, we may form the pull-back functors,

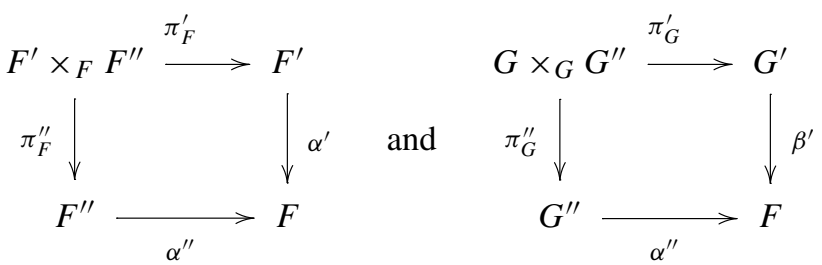

To simplify the notation, let $T=T_{\varepsilon}$ and $\eta=\eta_{\varepsilon}$. Observe that

$$
G^{\prime} T \times_{G T} G^{\prime \prime} T=\left(G^{\prime} \times_{G} G^{\prime \prime}\right) T,
$$

and so from the universal property of pull-backs we obtain the natural transformation

$$
\Phi=\varphi^{\prime} \times_{\varphi} \varphi^{\prime \prime}: F^{\prime} \times_{F} F^{\prime \prime} \rightarrow\left(G^{\prime} \times_{G} G^{\prime \prime}\right) T .
$$

Similarly, we get a natural transformation

$$
\Psi=\psi^{\prime} \times \psi_{\psi}^{\prime \prime}: G^{\prime} \times_{G} G^{\prime \prime} \rightarrow\left(F^{\prime} \times_{F} F^{\prime \prime}\right) T .
$$

We need to check that $\left(F^{\prime} \times{ }_{F} F^{\prime \prime}, G^{\prime} \times{ }_{G} G^{\prime \prime}, \Phi, \Psi\right)$ is an $\varepsilon$-interleaving that is indeed the relevant pull-back.

To see that we have an interleaving, it only remains to show that $(\Phi T) \Psi=\left(G^{\prime} \times_{G}\right.$ $\left.G^{\prime \prime}\right) \eta^{2}$ and $(\Psi T) \Phi=\left(F^{\prime} \times{ }_{F} F^{\prime \prime}\right) \eta^{2}$. We prove the second identity. The verification of the first is symmetric.

We observe that $(\Psi T) \Phi$ is one morphism $F^{\prime} \times{ }_{F} F^{\prime \prime} \rightarrow\left(F^{\prime} \times{ }_{F} F^{\prime \prime}\right) T^{2}$ that provides the unique dotted arrow (by the universal property of the pull-back) making the diagram

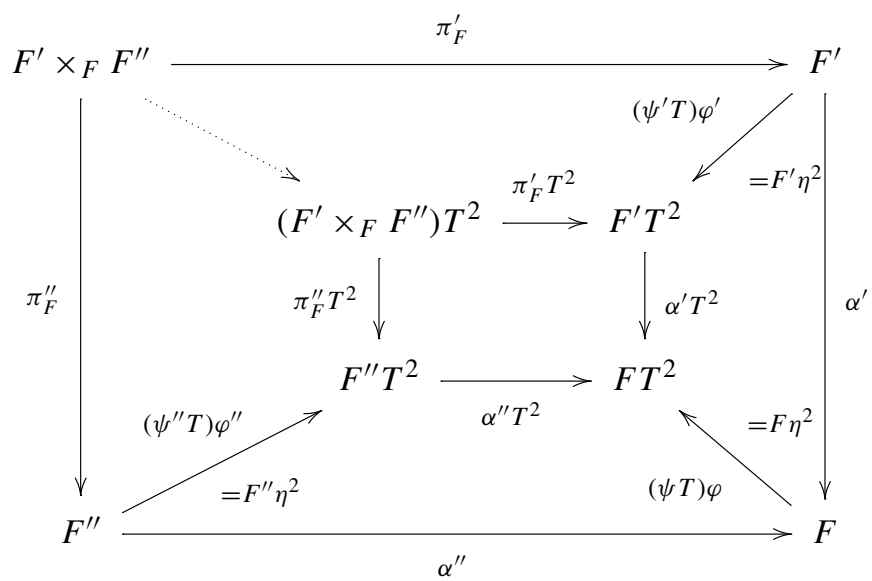


commute. Since $\left(F^{\prime} \times{ }_{F} F^{\prime \prime}\right) \eta^{2}$ also fits the diagram, by uniqueness, $(\Psi T) \Phi=$ $\left(F^{\prime} \times_{F} F^{\prime \prime}\right) \eta^{2}$.

Corollary 7.5 The category $\operatorname{Int}_{\varepsilon}(\mathbf{A})$ has all finite products and coproducts. Every morphism in $\mathbf{I n t}_{\varepsilon}(\mathbf{A})$ has a kernel and a cokernel.

Proof Since $\mathbf{I n t}_{\varepsilon}(\mathbf{A})$ has a terminal object and pull-backs, it has finite products. Since the category has an initial object and push-outs, it has finite coproducts. Since $\operatorname{Int}_{\varepsilon}(\mathbf{A})$ has a zero object and pull-backs, and the kernel of $(\alpha, \beta)$ : $(F, G, \varphi, \psi) \rightarrow\left(F^{\prime}, G^{\prime}, \varphi^{\prime}, \psi^{\prime}\right)$ can be obtained by pulling back along the initial morphism $(O, O, \omega, \omega) \rightarrow\left(F^{\prime}, G^{\prime}, \varphi^{\prime}, \psi^{\prime}\right)$, every morphism has a kernel. Similarly, every morphism has a cokernel since $\operatorname{Int}_{\varepsilon}(\mathbf{A})$ has a zero object and push-outs.

It remains to show that every monomorphism is a kernel and that every epimorphism is a cokernel. Before doing so, we show that in a preadditive category, monomorphisms and epimorphisms are characterized by their trivial kernels and cokernels, respectively.

Lemma 7.6 [19] Let $\mathbf{C}$ be a category with zero object, kernels, and cokernels. If $f: X \rightarrow Y$ is a monomorphism in $\mathbf{C}$, then $\operatorname{ker} f=0$. Dually, if $f$ is an epimorphism, then coker $f=0$.

If the category is preadditive, then we have the following converse for Lemma 7.6.

Lemma 7.7 Let $\mathbf{C}$ be a preadditive category with zero object, kernels, and cokernels. If $f: X \rightarrow Y$ is a morphism in $\mathbf{C}$ with trivial kernel, then $f$ is a monomorphism. Dually, if $f$ has trivial cokernel, then $f$ is an epimorphism.

Proof Suppose that ker $f=0$ and that $g, h: W \rightarrow X$ are morphisms that satisfy $f g=f h$. Then using the preadditive structure of $\mathbf{C}$, we find that $f(g-h)=0$, and so $g-h$ factors through ker $f=0$. It follows that $g-h=0$, so $g=h$. Therefore, $f$ is a monomorphism.

The proof of the dual statement is dual.

Next, we show that the above characterization of monomorphisms and epimorphisms applies to our setting.

Lemma 7.8 The category $\operatorname{Int}_{\varepsilon}(\mathbf{A})$ is preadditive.

Proof Let $X=(F, G, \varphi, \psi)$ and $X^{\prime}=\left(F^{\prime}, G^{\prime}, \varphi, \psi\right)$ be $\varepsilon$-interleavings. By definition, $\mathbf{I n t}_{\varepsilon}(\mathbf{A})(X, Y) \subset \mathbf{A}^{(\mathbb{R}, \leq)}\left(F, F^{\prime}\right) \times \mathbf{A}^{(\mathbb{R}, \leq)}\left(G, G^{\prime}\right)$, which is itself an abelian group. One can readily verify that $(0,0) \in \mathbf{I n t}_{\varepsilon}(\mathbf{A})(X, Y)$. Since $\mathbf{A}^{(\mathbb{R}, \leq)}$ is preadditive, composition distributes over the addition of morphisms. If $(\alpha, \beta),\left(\alpha^{\prime}, \beta^{\prime}\right) \in$ Int $_{\varepsilon}(\mathbf{A})(X, Y)$, then $\varphi^{\prime}\left(\alpha+\alpha^{\prime}\right)=\varphi^{\prime} \alpha+\varphi^{\prime} \alpha^{\prime}=(\beta T) \varphi+\left(\beta^{\prime} T\right) \varphi=\left(\left(\beta+\beta^{\prime}\right) T\right) \varphi$, so $\left(\alpha+\alpha^{\prime}, \beta+\beta^{\prime}\right) \in \operatorname{Int}_{\varepsilon}(\mathbf{A})(X, Y)$. Finally, we verify that if $(\alpha, \beta) \in \operatorname{Int}_{\varepsilon}(\mathbf{A})(X, Y)$, 
then so is its additive inverse. Since $\mathbf{A}^{(\mathbb{R}, \leq)}$ is preadditive, we have natural transformations $-\alpha$ and $-\beta$. Since $0=\varphi^{\prime} 0=\varphi^{\prime}(\alpha+(-\alpha))=\varphi^{\prime} \alpha+\varphi^{\prime}(-\alpha)$, it follows that $\varphi^{\prime}(-\alpha)=-\varphi^{\prime} \alpha$. Similarly, $(-\beta) \varphi=-\beta \varphi$. It follows that $(-\alpha,-\beta) \in$ $\operatorname{Int}_{\varepsilon}(\mathbf{A})(X, Y)$. Since $(\alpha, \beta)+(-\alpha,-\beta)=(0,0), \operatorname{Int}_{\varepsilon}(\mathbf{A})(X, Y)$ is an abelian group.

Composition is bilinear since it is the restriction of composition in the additive category $\mathbf{A}^{(\mathbb{R}, \leq)} \times \mathbf{A}^{(\mathbb{R}, \leq)}$.

Lemma 7.9 In $\mathbf{I n t}_{\varepsilon}(\mathbf{A})$, every monomorphism is a kernel, and every epimorphism is a cokernel.

Proof Let $(\alpha, \beta):(F, G, \varphi, \psi) \rightarrow\left(F^{\prime}, G^{\prime}, \varphi, \psi\right)$ be a monomorphism in $\operatorname{Int}_{\varepsilon}(\mathbf{A})$. We will show that $(\alpha, \beta)$ is the kernel of the natural morphism

$$
\pi:\left(F^{\prime}, G^{\prime}, \varphi, \psi\right) \rightarrow \operatorname{coker}(\alpha, \beta) .
$$

First, we calculate $\operatorname{ker}(\alpha, \beta)$ in terms of $\operatorname{ker} \alpha$ and $\operatorname{ker} \beta$. If we pull back the morphism $(\alpha, \beta):(F, G, \varphi, \psi) \rightarrow\left(F^{\prime}, G^{\prime}, \varphi^{\prime}, \psi^{\prime}\right)$ along the initial morphism $(O, O, \omega, \omega) \rightarrow$ $\left(F^{\prime}, G^{\prime}, \varphi^{\prime}, \psi^{\prime}\right)$, we obtain the interleaving $(\operatorname{ker} \alpha, \operatorname{ker} \beta, \Phi, \Psi)$ constructed in the proof of Lemma 7.4.

Cokernels are obtained in a dual manner; we have that $\operatorname{coker}(\alpha, \beta)=(\operatorname{coker} \alpha$, $\operatorname{coker} \beta, \bar{\Phi}, \bar{\Psi})$.

By Lemma 7.6, every monomorphism has trivial kernel. Thus, $\operatorname{ker}(\alpha, \beta)=$ $(\operatorname{ker} \alpha, \operatorname{ker} \beta, \Phi, \Psi)=(O, O, \omega, \omega)$. This means, in particular, that $\operatorname{ker} \alpha=$ $\operatorname{ker} \beta=O$. It follows from Lemmas 7.7 and 7.8 that $\alpha$ and $\beta$ are monomorphisms. Since $\mathbf{A}^{(\mathbb{R}, \leq)}$ is abelian, $\alpha$ is the kernel of the quotient map, $F^{\prime} \rightarrow \operatorname{coker} \alpha$, and likewise $\beta$ is the kernel of $G^{\prime} \rightarrow \operatorname{coker} \beta$. It then follows that $(\alpha, \beta)$ is the kernel of the natural morphism, $\left(F^{\prime}, G^{\prime}, \Phi, \Psi\right) \rightarrow(\operatorname{coker} \alpha, \operatorname{coker} \beta, \bar{\Phi}, \bar{\Psi})$.

The dual statement follows from the dual proof.

Combining Lemma 7.3, Corollary 7.5, and Lemma 7.9, we have the following.

Theorem 7.10 Given an abelian category $\mathbf{A}$ and $\varepsilon \geq 0$, the category $\operatorname{Int}_{\varepsilon}(\mathbf{A})$ of $\varepsilon$ interleavings in $\mathbf{A}$ is an abelian category.

From Theorem 7.10 and Lemma 7.4 we immediately have the following two applications.

Corollary 7.11 If the two pairs of diagrams $(F, G)$ and $\left(F^{\prime}, G^{\prime}\right)$ in $\mathbf{A}^{(\mathbb{R}, \leq)}$ are $\varepsilon$ interleaved, then so is the pair $\left(F \oplus F^{\prime}, G \oplus G^{\prime}\right)$.

Corollary 7.12 Let $(\alpha, \beta)$ be a morphism in $\mathbf{I n t}_{\varepsilon}(\mathbf{A})$. Then each of the following three pairs of diagrams in $\mathbf{A}^{(\mathbb{R}, \leq)}$ are $\varepsilon$-interleaved: $(\operatorname{ker} \alpha, \operatorname{ker} \beta),(\operatorname{im} \alpha, \operatorname{im} \beta)$, and (coker $\alpha, \operatorname{coker} \beta)$.

As an application of Corollary 7.12, we get the following generalization of the Stability Theorem of [9]. 
Theorem 7.13 (Stability Theorem for Kernels, Images, and Cokernels) Let $h: Y \rightarrow$ $X$ be a continuous map of topological spaces. Let $f, f^{\prime}: X \rightarrow \mathbb{R}$ and $g, g^{\prime}: Y \rightarrow \mathbb{R}$ be (not necessarily continuous) maps such that

$$
\text { for all } y \in Y, \quad f h(y) \leq g(y), \quad \text { and } \quad f^{\prime} h(y) \leq g^{\prime}(y) .
$$

Let $F \in \mathbf{T o p}^{(\mathbb{R}, \leq)}$ be given by $F(a)=f^{-1}(-\infty, a]$ and inclusion. Define $F^{\prime}, G$, and $G^{\prime}$ similarly. By (5), $h$ induces maps $\alpha: G \rightarrow F$ and $\beta: G^{\prime} \rightarrow F^{\prime}$ in $\mathbf{T o p}^{(\mathbb{R}, \leq)}$. Let $\mathbf{A}$ be an abelian category and $H: \mathbf{T o p} \rightarrow \mathbf{A}$ be some functor. Let $\varepsilon=\max \left\{\left\|f-f^{\prime}\right\|_{\infty}\right.$, $\left.\left\|g-g^{\prime}\right\|_{\infty}\right\}$. Then

$$
d(\operatorname{ker} H \alpha, \operatorname{ker} H \beta), \quad d(\operatorname{im} H \alpha, \operatorname{im} H \beta), \quad d(\operatorname{coker} H \alpha, \operatorname{coker} H \beta) \leq \varepsilon .
$$

Proof By the definition of $\varepsilon, F \hookrightarrow F^{\prime} T_{\varepsilon}, F^{\prime} \hookrightarrow F T_{\varepsilon}, G \hookrightarrow G^{\prime} T_{\varepsilon}$, and $G^{\prime} \hookrightarrow G T_{\varepsilon}$ in Top $^{(\mathbb{R}, \leq)}$. Since $\alpha$ and $\beta$ are both induced by $h$, the diagrams

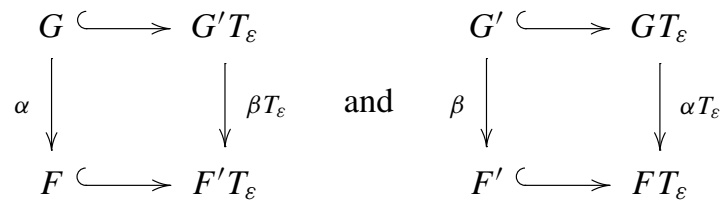

commute, and thus $(\alpha, \beta) \in \mathbf{I n t}_{\varepsilon}$ (Top). By functoriality $(H \alpha, H \beta) \in \mathbf{I n t}_{\varepsilon}(\mathbf{A})$ (see Proposition 7.2). Apply Corollary 7.12 and Definition 3.2 to obtain the desired result.

Strengthening (5), we obtain an extended persistence version of this theorem. It has essentially the same proof, so we omit it.

Theorem 7.14 (Stability Theorem for Kernels, Images, and Cokernels in Extended Persistence) Let $h: Y \rightarrow X$ be a continuous map of topological spaces. Let $f, f^{\prime}$ : $X \rightarrow(-\infty, M]$ be (not necessarily continuous) maps. Let $g=f h$ and $g^{\prime}=f^{\prime} h$. Let $s>0$. Let $F \in$ Pair $^{(\mathbb{R}, \leq)}$ be given by $F(c)=\left(f^{-1}(-\infty, c], \emptyset\right)$ if $c<b+s$ and $F(c)=\left(X, f^{-1}[2 b+s-c, \infty)\right)$ if $c \geq b+s$, and inclusion. Define $F^{\prime}, G$, and $G^{\prime}$ similarly. Then $h$ induces maps $\alpha: G \rightarrow F$ and $\beta: G^{\prime} \rightarrow F^{\prime}$ in Pair ${ }^{(\mathbb{R}, \leq)}$. Let $\mathbf{A}$ be an abelian category, and $H:$ Pair $\rightarrow \mathbf{A}$ be some functor. Then

$$
\begin{aligned}
& d(\operatorname{ker} H \alpha, \operatorname{ker} H \beta), \quad d(\operatorname{im} H \alpha, \operatorname{im} H \beta), \\
& d(\text { coker } H \alpha, \text { coker } H \beta) \leq\left\|f-f^{\prime}\right\|_{\infty} .
\end{aligned}
$$

\section{Future Work}

In this paper, we have studied persistence by considering diagrams indexed by $(\mathbb{R}, \leq)$. However, there are versions of persistence in which the objects of study can be viewed as diagrams with more general indexing categories. For example, we would like to 
be able to consider diagrams indexed by $\left(\mathbb{R}^{n}, \leq\right)$ for multidimensional persistence, $S^{1}$ for circle-valued persistence, and the category $\cdot \rightarrow \cdot \leftarrow \cdot \rightarrow \cdots \leftarrow$. for zig-zag persistence. This generalization will be presented in [1].

It would be nice to have a categorical definition of bottleneck distance arbitrary $(\mathbb{R}, \leq)$-indexed diagrams of vector spaces and to have a corresponding Isometry Theorem.

Acknowledgements The first author would like to thank Vin de Silva, Robert Ghrist, David Lipsky, John Oprea, and Radmila Sazdanovic for helpful conversations. Both authors thank Peter Landweber for many helpful corrections. The first author also gratefully acknowledges the support from AFOSR grant \# FA9550-13-1-0115.

\section{References}

1. Bubenik, P., de Silva, V., Scott, J.A.: Metrics for generalized persistence modules (2013). arXiv: 1312.3829

2. Cagliari, F., Ferri, M., Pozzi, P.: Size functions from a categorical viewpoint. Acta Appl. Math. 67(3), 225-235 (2001)

3. Carlsson, G.: Topology and data. Bull. Am. Math. Soc. (N.S.) 46(2), 255-308 (2009)

4. Carlsson, G., de Silva, V.: Zigzag persistence. Found. Comput. Math. 10(4), 367-405 (2010)

5. Chazal, F., Cohen-Steiner, D., Glisse, M., Guibas, L.J., Oudot, S.Y.: Proximity of persistence modules and their diagrams. In: Proceedings of the 25th Annual Symposium on Computational Geometry, SCG'09, pp. 237-246. ACM, New York (2009)

6. Chazal, F., de Silva, V., Glisse, M., Oudot, S.: The structure and stability of persistence modules (2012). arXiv:1207.3674

7. Cohen-Steiner, D., Edelsbrunner, H., Harer, J.: Stability of persistence diagrams. Discrete Comput. Geom. 37(1), 103-120 (2007)

8. Cohen-Steiner, D., Edelsbrunner, H., Harer, J.: Extending persistence using Poincaré and Lefschetz duality. Found. Comput. Math. 9(1), 79-103 (2009)

9. Cohen-Steiner, D., Edelsbrunner, H., Harer, J., Morozov, D.: Persistent homology for kernels, images, and cokernels. In: Proceedings of the Twentieth Annual ACM-SIAM Symposium on Discrete Algorithms, pp. 1011-1020. SIAM, Philadelphia (2009)

10. Crawley-Boevey, W.: Decomposition of pointwise finite-dimensional persistence modules (2012). arXiv: 1210.0819

11. Edelsbrunner, H., Harer, J.: Persistent homology—a survey. In: Surveys on Discrete and Computational Geometry. Contemp. Math., vol. 453, pp. 257-282. Am. Math. Soc., Providence (2008)

12. Edelsbrunner, H., Harer, J.L.: Computational Topology. Am. Math. Soc., Providence (2010). An introduction

13. Edelsbrunner, H., Letscher, D., Zomorodian, A.: Topological persistence and simplification. Discrete Comput. Geom. 28(4), 511-533 (2002). Discrete and computational geometry and graph drawing (Columbia, SC, 2001)

14. Freyd, P.: Abelian Categories. An Introduction to the Theory of Functors. Harper's Series in Modern Mathematics. Harper \& Row, New York (1964). xi+164

15. Ghrist, R.: Barcodes: the persistent topology of data. Bull. Am. Math. Soc. (N.S.) 45(1), 61-75 (2008)

16. Hungerford, T.W.: Algebra. Graduate Texts in Mathematics, vol. 73. Springer, New York (1980). Reprint of the 1974 original

17. Lesnick, M.: Multidimensional interleavings and applications to topological inference (2012). arXiv: 1106.5305

18. Mac Lane, S.: Categories for the Working Mathematician, 2nd edn. Graduate Texts in Mathematics, vol. 5. Springer, New York (1998)

19. Mitchell, B.: Theory of Categories. Pure and Applied Mathematics, vol. XVII. Academic Press, New York (1965)

20. Zomorodian, A.J.: Topology for Computing. Cambridge Monographs on Applied and Computational Mathematics, vol. 16. Cambridge University Press, Cambridge (2005)

21. Zomorodian, A., Carlsson, G.: Computing persistent homology. Discrete Comput. Geom. 33(2), 249274 (2005) 